\title{
Integrated Feedback Scheduling and Control Codesign for Motion Coordination of Networked Induction Motor Systems
}

\author{
Dezong Zhao, ${ }^{1}$ Qingqing Ding, ${ }^{2}$ Shangmin Zhang, ${ }^{3}$ Chunwen $\mathrm{Li}^{3}{ }^{3}$ and Richard Stobart ${ }^{1}$ \\ ${ }^{1}$ Department of Aeronautical and Automotive Engineering, Loughborough University, Loughborough LE11 3TU, UK \\ ${ }^{2}$ Department of Electrical Engineering, Tsinghua University, Beijing 100084, China \\ ${ }^{3}$ Department of Automation, Tsinghua University, Beijing 100084, China
}

Correspondence should be addressed to Dezong Zhao; d.zhao2@lboro.ac.uk

Received 31 May 2013; Revised 28 January 2014; Accepted 29 January 2014; Published 16 March 2014

Academic Editor: M. Onder Efe

Copyright (C) 2014 Dezong Zhao et al. This is an open access article distributed under the Creative Commons Attribution License, which permits unrestricted use, distribution, and reproduction in any medium, provided the original work is properly cited.

\begin{abstract}
This paper investigates the codesign of remote speed control and network scheduling for motion coordination of multiple induction motors through a shared communication network. An integrated feedback scheduling algorithm is designed to allocate the optimal sampling period and priority to each control loop to optimize the global performance of a networked control system (NCS), while satisfying the constraints of stability and schedulability. A speed synchronization method is incorporated into the scheduling algorithm to improve the speed synchronization performance of multiple induction motors. The rational gain of the network speed controllers is calculated using the Lyapunov theorem and tuned online by fuzzy logic to guarantee the robustness against complicated variations on the communication network. Furthermore, a state predictor is designed to compensate the time delay which occurred in data transmission from the sensor to the controller, as a part of the networked controller. Simulation results support the effectiveness of the proposed control-and-scheduling codesign approach.
\end{abstract}

\section{Introduction}

The applications of NCSs have been an important trend in modern industry owing to the convenient remote operation and cost-effective installation. In such systems, spatially distributed sensors, actuators, and controllers share information through the network instead of complex wiring, resulting in flexible and open architecture. NCSs have been found to be applications in a broad range of areas such as mobile robots $[1-5]$, unmanned aerial vehicles $[6-8]$, remote surgery $[9,10]$, and mining systems [11]. Considering the common grounds that they are driven by electrical motors and communicate via network, such systems are called networked motion control systems (NMCSs) [12]. NMCSs are constructed on the basis of remote motion controller and local motor drivers, using network to realize transmission of control orders and motion states. NMCSs are hot research topics of NCSs and play important roles in factory automation. Most of the current NMCSs focus on networked DC motor control [13, 14], for DC motor being an ideal networked control plant with linear model. Actually, induction motors play a dominant part in industrial applications for their merits of simple structure and high reliability. However, networked induction motor control is rather more complicated due to the nonlinear dynamics of induction motors [15-17]. Networked induction motor control is a rather challenging research topic.

New concepts of operation bring new notions in the control system, including the quality of service (QoS), link, and configuration. Time delay and packets dropout are the two most important issues to be concerned which would result in NCSs performance deterioration and potential system instability [18]. It is particularly important in dealing with the two issues in designing networked motion controllers, such as gain scheduling and sampling period adaptation, for NMCSs being time critical due to their fast dynamics. The NCS control strategies can be grouped into two categories: stability analysis based methods [19-21] and system synthesis methods [22-24]. In stability analysis based methods, the NCS controllers are designed primarily with the assumption of no information lost and then analyze the system performance considering the network environment. The system synthesis 
methods are more practical, where the controller parameters and sampling periods are obtained with the consideration of communication constraints.

On the other hand, the overall performance of a multipleloop NCS depends on both of the control algorithm and scheduling algorithm. The traditional static scheduling methods cannot find the optimal solution of the NCS for the sampling period and priority of each loop being calculated offline [25]. Considering the tradeoff between the quality of service (QoS) of the network and the quality of control (QoC) of the NCS, the codesign of network controller and scheduling method is an efficient way [26-28]. In the codesign method, the scheduling algorithm updates the sampling period and priority of each loop online, such that the global optimization of the NCS is approached. As an illustration, the codesign approach for the motion coordination of multiple induction motors in a NMCS is shown in Figure 1, where $A_{i}, C_{i}, S_{i}$, and $P_{i}$ denote the actuator, controller, sensor, and plant in loop $i$, respectively.

In this paper, an integrated feedback scheduling strategy is proposed, including the optimal bandwidth allocation scheme, online priority modification scheme, and adjacent cross coupling control structure. An optimization problem is formulated as minimizing the sum of the tracking error of each control loop, with the constraints of stability and available network bandwidth, to improve the speed synchronization performance of the NMCS. In designing the networked speed controller, its rational gain is calculated using the Lyapunov theorem and tuned online by fuzzy logic.

The paper is organized as follows. After the introduction in Section 1, the system description is presented in Section 2. The networked speed controller is proposed in Section 3. The integrated feedback scheduling strategy is presented in Section 4. The simulation results are stated in Section 5. Finally, the conclusions are summarized in Section 6.

\section{System Description}

The structure diagram of the investigated NMCS is shown in Figure 2 in more detail, where the bandwidth-limited control network is shared by $N$ control loops therein. In the NMCS, a priority-driven medium access control (MAC) protocol is employed, such as the DeviceNet. According to the nonpreemptive scheduling standard, each loop is assigned with a unique priority. In loop $i$, the output speed of motor $P_{i}$ is sampled by the sensor $S_{i}$ with the sampling period of $h_{i}$ and sent to the controller $C_{i}$ with the priority $p_{i}$. A computer or a node in the application layer behaves as the master node to perform the integrated feedback scheduling algorithm. In the decision-making process, $h_{i}$ and $p_{i}$ are updated according to the QoS and the feedback speed of all loops.

In the proposed codesign methodology, the following assumptions are made: (1) the sensor is time-driven; (2) the controller and the actuator are event-driven; and (3) the data sampled in one period can be encapsulated and transmitted in one packet.

As shown in Figure 3, the components of each control loop can be grouped into five modules: (1) the induction

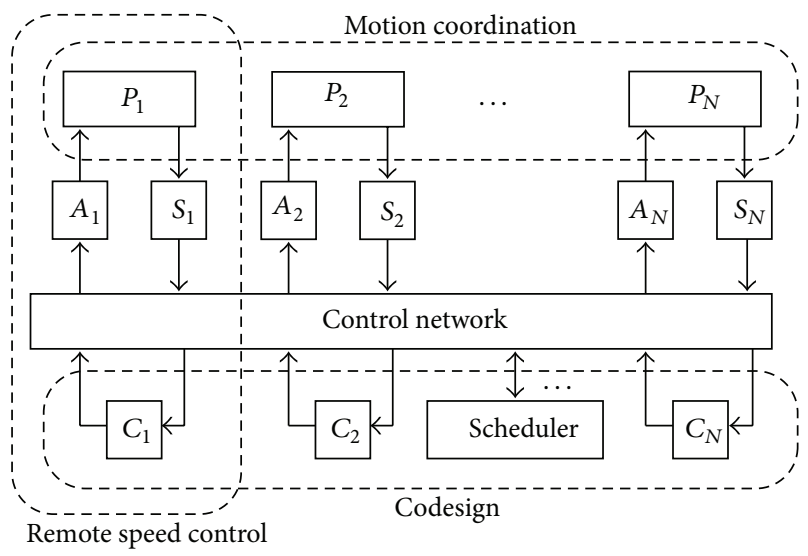

FIGURE 1: Codesign of control and scheduling algorithms for motion coordination of a NMCS.

motor and the sensor; (2) the communication network; (3) the networked controller; (4) the actuator; and (5) the local controller, which are described in the following subsections, respectively. The reference speed is denoted as $\omega^{*}$.

2.1. Induction Motor and the Sensor. The dynamics of a threephase squirrel induction motor in the stator fixed $\alpha-\beta$ reference frame is described as the following differential equations [29]:

$$
\begin{gathered}
\dot{i}_{\alpha s}=-\gamma i_{\alpha s}+\alpha \beta \psi_{\alpha r}+n_{p} \beta \omega \psi_{\beta r}+\frac{u_{\alpha s}}{\left(\sigma L_{s}\right)}, \\
\dot{i}_{\beta s}=-\gamma i_{\beta s}+\alpha \beta \psi_{\beta r}-n_{p} \beta \omega \psi_{\alpha r}+\frac{u_{\beta s}}{\left(\sigma L_{s}\right)}, \\
\dot{\psi}_{\alpha r}=\alpha M i_{\alpha s}-\alpha \psi_{\alpha r}-n_{p} \omega \psi_{\beta r}, \\
\dot{\psi}_{\beta r}=\alpha M i_{\beta s}-\alpha \psi_{\beta r}+n_{p} \omega \psi_{\alpha r}, \\
\dot{\omega}=\mu\left(\psi_{\alpha r} i_{\beta s}-\psi_{\beta r} i_{\alpha s}\right)-\frac{\left(T_{L}+K_{f} \omega\right)}{J},
\end{gathered}
$$

where the two-dimensional vectors $i_{s}=\left[\begin{array}{ll}i_{\alpha s} & i_{\beta s}\end{array}\right]^{T}, \psi_{r}=$ $\left[\begin{array}{ll}\psi_{\alpha r} & \psi_{\beta r}\end{array}\right]^{T}$, and $u=\left[\begin{array}{ll}u_{\alpha s} & u_{\beta s}\end{array}\right]^{T}$ are the stator currents, rotor fluxes, and stator voltages, respectively. $\omega$ is the mechanical rotor speed; $R_{s}$ and $R_{r}$ are the stator and rotor resistances, respectively; $L_{s}$ and $L_{r}$ are the stator and rotor self-inductances, respectively; $M$ is the stator-rotor mutual inductance; $T_{L}$ is the load torque; $K_{f}$ is the friction coefficient; $J$ is the motor-load moment of inertia; and $n_{p}$ is the number of pole pairs. Denote the leakage factor by $\sigma=1-$ $M^{2} /\left(L_{s} L_{r}\right)$, the rotor time constant by $T_{r}=L_{r} / R_{r}$, and the other parameters by $\alpha=1 / T_{r}, \beta=M /\left(\sigma L_{s} L_{r}\right), \gamma=$ $M^{2} R_{r} /\left(\sigma L_{s} L_{r}^{2}\right)+R_{s} /\left(\sigma L_{s}\right)$, and $\mu=3 n_{p} M /\left(2 J L_{r}\right)$. The mechanical equation (le) can be expressed in terms of the electromagnetic torque $T_{e}$ :

$$
T_{e}=J \dot{\omega}+K_{f} \omega+T_{L} .
$$




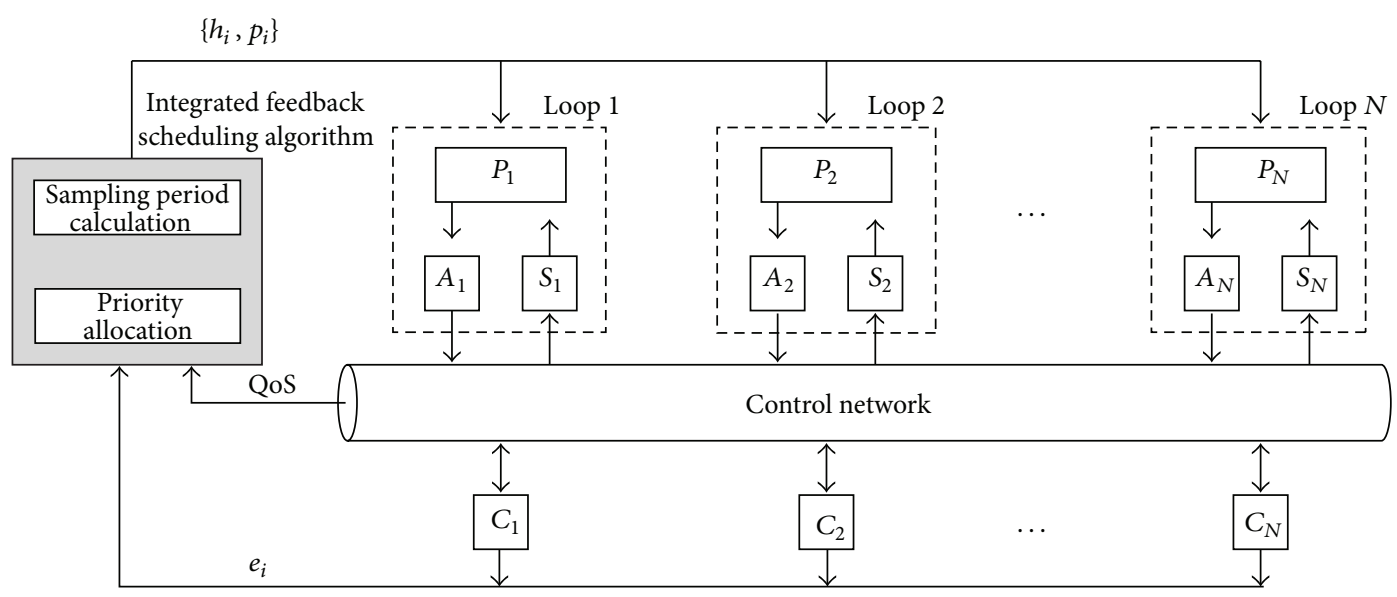

FIGURE 2: Structure of the investigated NMCS.

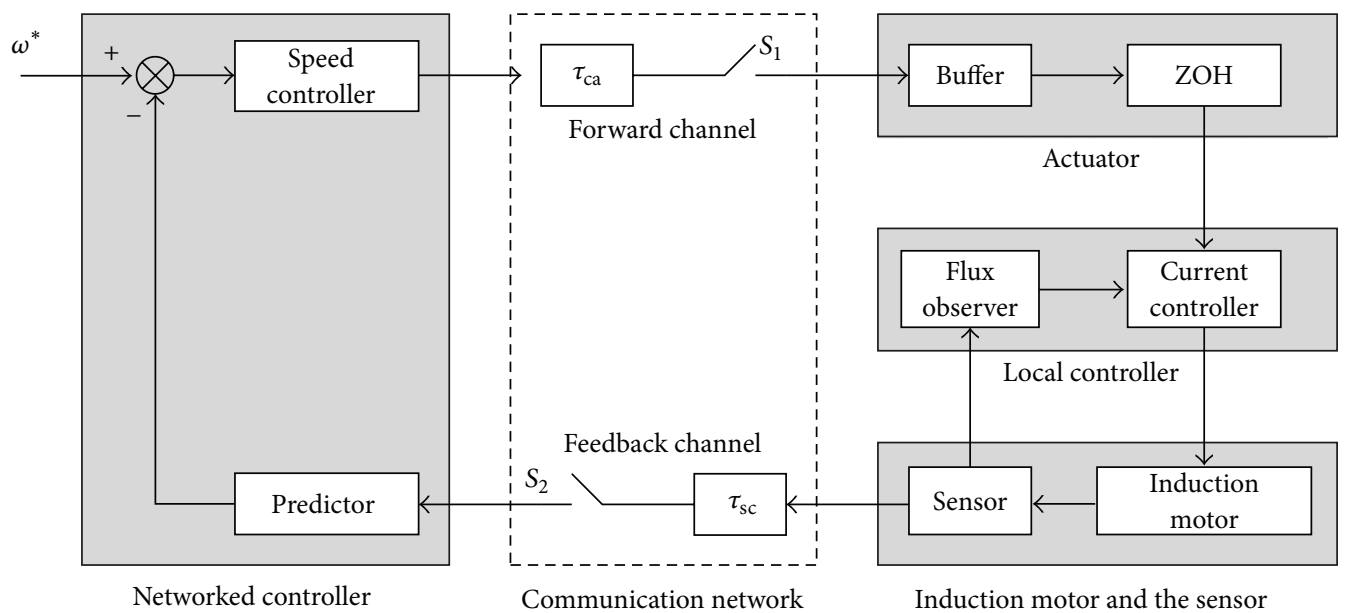

FIgURE 3: Structure of a single control loop in the NMCS.

The induction motor speed is measured by the sensor periodically and is sent to the networked controller via the network together with its time stamp.

2.2. Communication Network. The network-induced delay consists of the sensor-to-controller delay $\tau_{\mathrm{sc}}$ and the controller-to-actuator delay $\tau_{\mathrm{ca}}$ and can be lumped together as $\tau=\tau_{\text {sc }}+\tau_{\text {ca }}$.

2.3. Networked Controller. The networked controller consists of two parts: a speed controller and a state predictor. A fuzzy logic PI controller is employed as the speed controller, where the gain values are tuned online by the fuzzy logic mechanism. The state predictor is designed in the feedback channel to compensate the negative impact brought by the feedback delay $\tau_{\text {sc }}$.

2.4. Actuator. The actuator is triggered when receiving data from the controller. The buffer size of the actuator is 1 , to guarantee that the latest control packet is used. Any newly arrived control packet at the actuator will update the control signal with older time stamp (if existing) in the buffer; otherwise, it will be discarded. At each sampling instant, the control command in the buffer is read by the zero-order hold $(\mathrm{ZOH})$ circuit and sent to the motor.

2.5. Local Controller. The local controller consists of the current regulator and the flux observer. A sliding mode estimator and a PI controller are adopted as the flux observer and current regulator, respectively. For more details, the readers can refer to [30] for more details. Using field orientation technique, the induction motor model is simplified as a DC motor linear model. The synchronous rotating angle of the rotor flux can be calculated from the estimated flux:

$$
\widehat{\theta}_{e}=\arctan \left(\frac{\widehat{\psi}_{\beta r}}{\widehat{\psi}_{\alpha r}}\right) \text {. }
$$

The stator currents under the synchronous rotating $d-q$ coordination are obtained by

$$
\left[\begin{array}{l}
i_{d s} \\
i_{q s}
\end{array}\right]=\left[\begin{array}{cc}
\cos \left(\hat{\theta}_{e}\right) & \sin \left(\widehat{\theta}_{e}\right) \\
-\sin \left(\widehat{\theta}_{e}\right) & \cos \left(\widehat{\theta}_{e}\right)
\end{array}\right]\left[\begin{array}{l}
i_{\alpha s} \\
i_{\beta s}
\end{array}\right],
$$


and the rotor fluxes $\widehat{\psi}_{q r}=0$ and $\widehat{\psi}_{d r}=\sqrt{\widehat{\psi}_{\alpha r}^{2}+\widehat{\psi}_{\beta r}^{2}}$ are satisfied under rotor field orientation. Accordingly, the mechanical equation (1e) can be represented as

$$
\dot{\omega}=\frac{K_{t}}{J} i_{q s}-\frac{K_{f}}{J} \omega-\frac{T_{L}}{J},
$$

where $K_{t}=\mu \widehat{\psi}_{d r}$.

\section{Problem Formulation}

For a NMCS including $N$ independent loops, the controlled plant of the loop $i$ can be expressed as

$$
\dot{x}_{p}^{i}(t)=A_{p}^{i} x_{p}^{i}(t)+B_{p}^{i} u_{p}^{i}(t), \quad y_{p}^{i}(t)=C_{p}^{i} x_{p}^{i}(t),
$$

with $x_{p}^{i}(t) \in \mathbb{R}^{N_{P}^{i}}, y_{p}^{i}(t) \in \mathbb{R}^{N_{s}^{i}}$, and $u_{p}^{i}(t) \in \mathbb{R}^{N_{A}^{i}}$, where $N_{P}^{i}, N_{S}^{i}$, and $N_{A}^{i}$ are the dimensions of the plant, sensor, and actuator of the loop $i$, respectively. $A_{p}^{i}, B_{p}^{i}$, and $C_{p}^{i}$ are coefficient matrices with proper dimensions.

The controller in the loop $i$ can be expressed as

$$
\begin{gathered}
\dot{x}_{c}^{i}(t)=A_{c}^{i} x_{c}^{i}(t)+B_{c}^{i} u_{c}^{i}(t), \\
y_{c}^{i}(t)=C_{c}^{i} x_{c}^{i}\left(t-\tau_{c}^{i}\right)+D_{c}^{i} u_{c}^{i}\left(t-\tau_{c}^{i}\right),
\end{gathered}
$$

with $x_{c}^{i}(t) \in \mathbb{R}^{N_{P}^{i}}, y_{c}^{i}(t) \in \mathbb{R}^{N_{A}^{i}}$, and $u_{c}^{i}(t) \in \mathbb{R}^{N_{s}^{i}}$, where $A_{c}^{i}, B_{c}^{i}$, $C_{c}^{i}$, and $D_{c}^{i}$ are coefficient matrices with proper dimensions. $\tau_{c}^{i}$ is the computational delay of the loop $i$ satisfying $0 \leq \tau_{c}^{i} \leq$ $\tau_{c \text {, max }}^{i}$, where $\tau_{c, \text { max }}^{i}$ is the maximum delay of the controller $i$. Generally speaking, $\tau_{c}^{i}$ is short and can be lumped into $\tau_{\mathrm{ca}}^{i}$.

Considering the delay on the feedback speed signal and forward control signal in the network, the following equations hold:

$$
u_{c}^{i}(t)=y_{p}^{i}\left(t-\tau_{\mathrm{sc}}^{i}\right), \quad u_{p}^{i}(t)=y_{c}^{i}\left(t-\tau_{\mathrm{ca}}^{i}\right),
$$

with $0 \leq \tau_{\mathrm{sc}}^{i} \leq \tau_{\mathrm{sc} \text { max }}^{i}$ and $0 \leq \tau_{\mathrm{ca}}^{i} \leq \tau_{\mathrm{ca} \text { max }}^{i}$, where $\tau_{\mathrm{sc}}^{i}$ and $\tau_{\mathrm{ca}}^{i}$ are the delay in the feedback channel and forward channel of the loop $i$, respectively; $\tau_{\mathrm{sc}, \max }^{i}$ and $\tau_{\mathrm{ca}, \max }^{i}$ are the upper limits of $\tau_{\mathrm{sc}}^{i}$ and $\tau_{\mathrm{ca}}^{i}$, respectively.

From (6)-(8), the closed loop state equation of the loop $i$ can be represented as

$$
\begin{aligned}
\dot{x}^{i}(t)= & {\left[\begin{array}{cc}
A_{p}^{i} & 0 \\
0 & A_{c}^{i}
\end{array}\right] x^{i}(t)+\left[\begin{array}{cc}
0 & 0 \\
B_{c}^{i} C_{c}^{i} & 0
\end{array}\right] x^{i}\left(t-\tau_{\mathrm{sc}}^{i}\right) } \\
& +\left[\begin{array}{cc}
B_{p}^{i} D_{c}^{i} C_{p}^{i} & 0 \\
0 & 0
\end{array}\right] x^{i}\left(t-\tau_{\mathrm{sc}}^{i}-\tau_{\mathrm{ca}}^{i}-\tau_{c}^{i}\right) \\
& +\left[\begin{array}{cc}
0 & B_{p}^{i} C_{c}^{i} \\
0 & 0
\end{array}\right] x^{i}\left(t-\tau_{\mathrm{ca}}^{i}-\tau_{c}^{i}\right),
\end{aligned}
$$

where $x^{i}(t)=\left[\left(x_{p}^{i}(t)\right)^{T}\left(x_{c}^{i}(t)\right)^{T}\right]^{T}$. Rewrite (9) as

$$
\begin{aligned}
\dot{x}^{i}(t)= & F^{i} x^{i}(t)+F_{1}^{i} x^{i}\left(t-\tau_{1}^{i}\right)+F_{2}^{i} x^{i}\left(t-\tau_{2}^{i}\right) \\
& +F_{3}^{i} x^{i}\left(t-\tau_{3}^{i}\right),
\end{aligned}
$$

where $F^{i}=\left[\begin{array}{cc}A_{p}^{i} & 0 \\ 0 & A_{c}^{i}\end{array}\right], F_{1}^{i}=\left[\begin{array}{cc}0 & 0 \\ B_{c}^{i} C_{c}^{i} & 0\end{array}\right], F_{2}^{i}=\left[\begin{array}{ccc}B_{p}^{i} D_{c}^{i} C_{p}^{i} & 0 \\ 0 & 0\end{array}\right], F_{3}^{i}=$ $\left[\begin{array}{cc}0 & B_{p}^{i} C_{c}^{i} \\ 0 & 0\end{array}\right], 0 \leq \tau_{1}^{i}=\tau_{\mathrm{sc}}^{i} \leq \tau_{\mathrm{sc}, \max }^{i}=\tau_{1, \max }^{i}, 0 \leq \tau_{2}^{i}=\tau_{\mathrm{sc}}^{i}+$ $\tau_{\mathrm{ca}}^{i}+\tau_{c}^{i} \leq \tau_{\mathrm{sc} \text {, max }}^{i}+\tau_{\mathrm{ca} \text {, max }}^{i}+\tau_{c, \text { max }}^{i}=\tau_{2, \text { max }}^{i}$, and $0 \leq \tau_{3}^{i}=$ $\tau_{\mathrm{ca}}^{i}+\tau_{c}^{i} \leq \tau_{\mathrm{ca} \text {, } \max }^{i}+\tau_{c, \text { max }}^{i}=\tau_{3, \text { max }}^{i}$. Each loop in the NMCS can be described by (10), where the time delay is sorted as $\tau_{1}^{i}, \tau_{2}^{i}$, and $\tau_{3}^{i}$. Therefore, the investigated NMCS can be represented as an augmented state space model:

$$
\begin{array}{r}
\dot{x}(t)=F x(t)+\sum_{i=1}^{N} F_{i} x\left(t-\tau_{i}\right), \quad x(t)=\phi(t), \\
t \in\left[\begin{array}{ll}
-\bar{\tau} & 0
\end{array}\right],
\end{array}
$$

where $x(t) \in \mathbb{R}^{N}$ is the system state, $\tau_{i}>0$ is the network delay, $\phi(\cdot)$ is the initial state, $F$ and $F_{i}$ are the coefficient matrices with proper dimensions, and $\bar{\tau}$ is the upper limit of $\tau_{i}$.

\section{Networked Speed Controller Design}

Considering the influence of the QoS variation on the control performance, fuzzy logic is adopted in gain adaptation of the networked speed controller. Furthermore, a state predictor placed is employed to minimize the trajectory deviation due to the time delay. The design process of the fuzzy speed controller and the state predictor in a control loop are introduced as follows.

4.1. Stability Analysis. In this section, the rational gain of the state feedback controller is to be selected using the Lyapunov method. Several criteria in time-delay systems are introduced in respect of the system stability.

Lemma 1 (see [31]). Assume that $a(\cdot) \in \mathbb{R}^{n_{a}}, b(\cdot) \in \mathbb{R}^{n_{b}}$, and $W(\cdot) \in \mathbb{R}^{n_{a} \times n_{b}}$ are defined on the interval $\Omega$. For any matrices $X \in \mathbb{R}^{n_{s} \times n_{s}}, Y \in \mathbb{R}^{n_{s} \times n_{b}}$, and $Z \in \mathbb{R}^{n_{b} \times n_{b}}$ satisfying $\left[\begin{array}{cc}X & Y \\ Y^{T} & Z\end{array}\right] \geq 0$, the following inequality holds:

$$
\begin{aligned}
& -2 \int_{\Omega} a^{T}(\alpha) W b(\alpha) d \alpha \\
& \quad \leq \int_{\Omega}\left[\begin{array}{l}
a(\alpha) \\
b(\alpha)
\end{array}\right]^{T}\left[\begin{array}{cc}
X & Y-W \\
Y^{T}-W^{T} & Z
\end{array}\right]\left[\begin{array}{l}
a(\alpha) \\
b(\alpha)
\end{array}\right] d \alpha .
\end{aligned}
$$

The Schur complement lemma can be transformed into the form of Riccati inequality.

Lemma 2 (see [32]). For the given constant matrices $\mathscr{A}$ and $Q=Q^{T}$, if there exists matrix variable $\mathscr{P}>0$ satisfying

$$
\left[\begin{array}{cc}
\mathscr{Q} & \mathscr{A} \\
\mathscr{A}^{T} & -\mathscr{P}^{-1}
\end{array}\right]<0
$$

then the following inequality holds:

$$
\mathscr{A} \mathscr{P} \mathscr{A}^{T}+Q<0 .
$$


The following theorem represents the delay-dependent stability condition of the NMCS.

Theorem 3. If there exist matrices $P>0, Q_{i}>0$, and $X_{i}, Y_{i}$, and $Z_{i}$ with proper dimensions such that

$$
\left[\begin{array}{cc}
\mathscr{F}_{11} & \mathscr{F}^{T} \mathscr{Z} \\
\mathscr{Z}^{T} \mathscr{F} & -\Gamma
\end{array}\right]<0, \quad\left[\begin{array}{cc}
X_{i} & Y_{i} \\
Y_{i}^{T} & Z_{i}
\end{array}\right] \geq 0,
$$

where

$$
\begin{gathered}
\mathscr{F}_{11} \triangleq\left[\begin{array}{cc}
\mathscr{H}_{11} & P \mathscr{F}_{1}-\mathscr{Y} \\
\mathscr{F}_{1}^{T} P^{T}-\mathscr{Y}^{T} & -\mathscr{L}
\end{array}\right], \\
\mathscr{F} \triangleq\left[\begin{array}{llll}
F & F_{1} & \cdots & F_{N}
\end{array}\right], \quad \mathscr{F}_{1} \triangleq\left[\begin{array}{lll}
F_{1} & \cdots & F_{N}
\end{array}\right], \\
\mathscr{Y} \triangleq\left[\begin{array}{lll}
Y_{1} & \cdots & Y_{N}
\end{array}\right], \quad \mathscr{Z} \triangleq \bar{\tau}\left[\begin{array}{lll}
Z_{1} & \cdots & Z_{N}
\end{array}\right], \\
\mathscr{H}_{11} \triangleq F^{T} P+P F+\sum_{i=1}^{N}\left\{Y_{i}+Y_{i}^{T}+\bar{\tau} X_{i}+Q_{i}\right\}, \\
\Gamma \triangleq \bar{\tau} \operatorname{diag}\left\{Z_{1}, \ldots, Z_{N}\right\},
\end{gathered}
$$

then the system (11) is asymptotically stable for any time delay $0 \leq \tau_{i} \leq \bar{\tau}$.

The proof is given in the appendix. Using Theorem 3, the rational range of the networked speed controller gain in each control loop can be obtained via the given $\bar{\tau}$. For $\bar{\tau}$ being normally a determined value under different network conditions, Theorem 3 gives the reference to set the original value of the controller gain.

4.2. Speed Controller Design. The fuzzy speed controller shown in Figure 4 comprises the fuzzy logic mechanism and the PI regulator. Figure 5 shows the membership functions of the input and output linguistic variables, where $E, P$, and $I$ denote the fuzzy values of $e, K_{P}$, and $K_{I}$, respectively, together with $k_{e}, g_{p}$, and $g_{i}$ indicating the membership bounds of them. The fuzzy control rules are defined in Table $1 . K_{P}$ and $K_{I}$ are initialized according to the no-delay system and are tuned online by the fuzzy inference according to the feedback speed error, such that the control command is updated to compensate the delay. The updating law of the gains is

$$
K_{P}^{\prime}=K_{P}+\Delta K_{P}, \quad K_{I}^{\prime}=K_{I}+\Delta K_{I},
$$

where $\Delta K_{P}$ and $\Delta K_{I}$ are the increment values of $K_{P}$ and $K_{I}$, respectively, while $K_{I}^{\prime}$ and $K_{I}^{\prime}$ are the updated gains. The initial value of $K_{P}$ should take the reference of Theorem 3, and the initial value of $K_{I}$ is given by a small constant.

4.3. State Predictor Design. $\tau_{\mathrm{sc}}$ and $\tau_{\mathrm{ca}}$ are different in nature where $\tau_{\mathrm{sc}}$ can be known when the controller uses the sensor data to generate the control signal, provided that the sensor

\begin{tabular}{|c|c|c|c|c|c|c|c|}
\hline & & & & $P$ & & & \\
\hline$E$ & NB & NM & NS & $\mathrm{ZO}$ & PS & PM & $\mathrm{PB}$ \\
\hline \multicolumn{8}{|l|}{ I } \\
\hline NS & NB & & & & & & \\
\hline NS & & NM & & & & & \\
\hline $\mathrm{ZO}$ & & & NS & & & & \\
\hline $\mathrm{ZO}$ & & & & $\mathrm{ZO}$ & & & \\
\hline $\mathrm{ZO}$ & & & & & PS & & \\
\hline PS & & & & & & $\mathrm{PM}$ & \\
\hline PS & & & & & & & $\mathrm{PB}$ \\
\hline
\end{tabular}
message is time-stamped. Therefore, a predictor can be used to estimate the available plant state in calculating the control law. However, $\tau_{\text {ca }}$ cannot be compensated using the time stamp method in decision making of control laws. The predictive control methods can be used to release the effect
TABLE 1: Fuzzy control rule base.

caused by $\tau_{\mathrm{ca}}$. The state predictor is used to compensate $\tau_{\mathrm{sc}}$, to obtain a more accurate plant state estimation. Considering $K_{f}$ is very little when the induction motor running in the constant power region, (5) can be expressed as

$$
\dot{\omega}=\frac{K_{t}}{J} i_{q s}-\frac{T_{L}}{J} ;
$$

thus the motor speed can be obtained by

$$
\omega(t)=\omega\left(t_{0}\right)+\frac{K_{t}}{J} \int_{t_{0}}^{t} i_{q s}(s) d s-\frac{T_{L}}{J}\left(t-t_{0}\right) .
$$

The timing diagram of the signals in NMCS considering the state prediction is shown in Figure 6, where $\tau_{k}$ denotes the lumped time delay in the sampling period $[k h,(k+1) h], \tau_{s c, k}$ denotes the sensor-to-controller delay for the sampled motor speed $\omega(k h), \tau_{\mathrm{ca}, k}$ denotes the controller-to-actuator delay for the control command $i_{q s}(k h)$, and $\widehat{\omega}\left(k h+\tau_{s c, k}\right)$ denotes the compensated feedback speed. The compensated speed signal within $[k h,(k+1) h]$ can be represented by the following discretized equation:

$$
\widehat{\omega}\left(k h+\tau_{\mathrm{scc}, k}\right)=\omega(k h)+\left(\frac{K_{t}}{J} i_{q s}(k h)-\frac{1}{J} T_{L}\right) \tau_{\mathrm{sc}, k} .
$$

\section{Integrated Feedback Scheduling}

In the proposed integrated feedback scheduling method, the sampling period and priority of each control loop are allocated under the constraints of stability and available network bandwidth, to realize the global optimization of the NMCS performance. The speed coupling error $e^{*}(t)$ is also calculated as a reference in calculating the control law; therefore, the motion coordination of multiple controlled induction motors is achieved. Denote the assigned bandwidth to the control loop $i$ by $b_{i}=c_{i} / h_{i}$, where $c_{i}$ and $h_{i}$ are the data processing time and sampling period, respectively. The schedulability criterion can refer to the sufficient condition in applying the RM scheduling strategy in a general NCS.

Lemma 4 (see [25]). For a NCS with N independent control loops, where a nonpreemptive control network is used, the NCS 


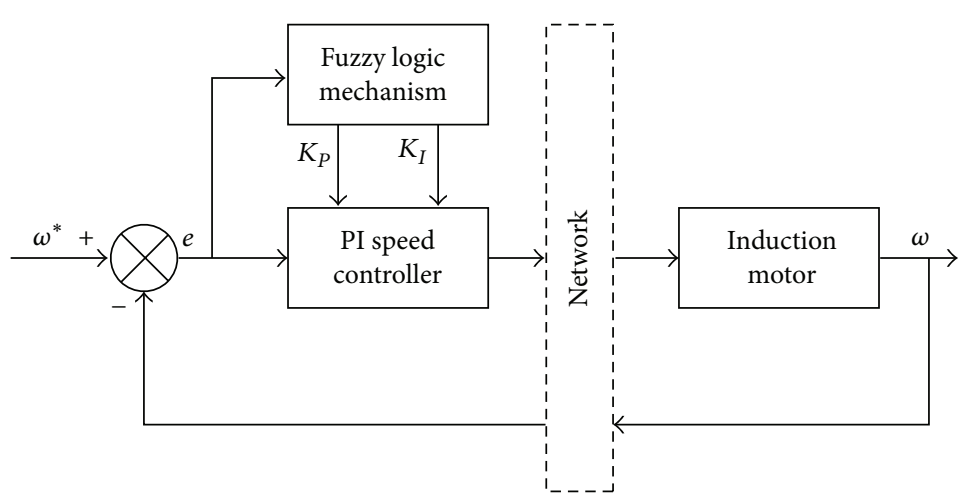

FIGURE 4: Networked fuzzy speed controller.

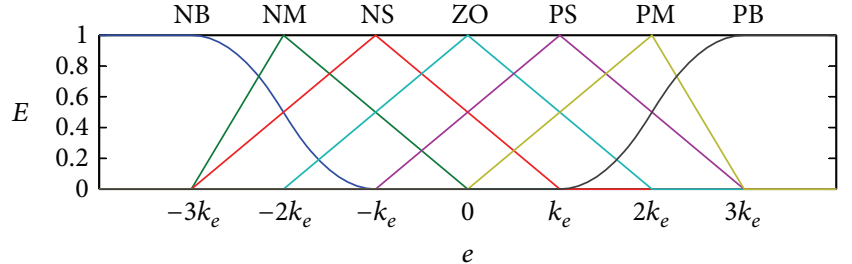

(a)

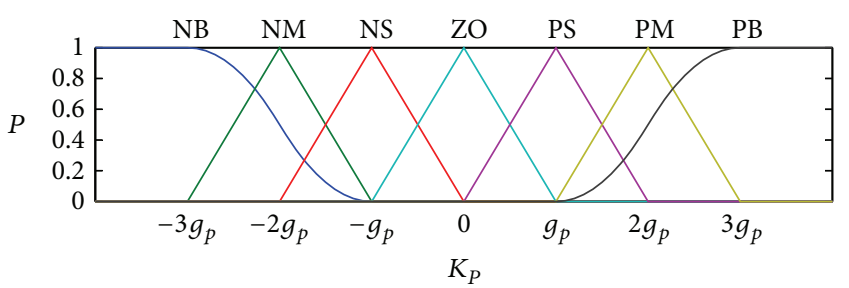

(b)

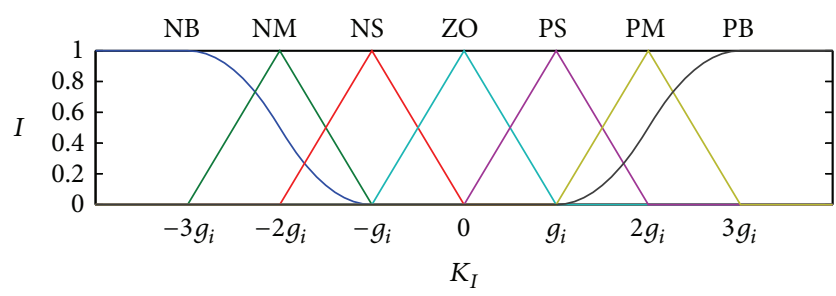

(c)

Figure 5: Membership functions of the input $e$ and outputs $K_{P}$ and $K_{I}$.

is schedulable with RM algorithm if (21) is satisfied for $i=$ $1, \ldots, N$ :

$$
b_{1}+b_{2}+\cdots+b_{i}+\frac{\bar{c}_{i}}{h_{i}} \leq i\left(2^{1 / i}-1\right)
$$

where $h_{1} \leq h_{1} \leq \cdots h_{n} ; \bar{c}_{i}$ is the worst-case blocking time of task $i$ by lower priority tasks; that is, $\bar{c}_{i}=\max _{j=i+1, \ldots, N} c_{j}$.

5.1. Optimal Sampling Period Assignment. The optimal sampling period assignment method is presented based on minimizing the transmission error between two contiguous sampling periods, with the constraints of stability and communications. The method is denoted as the optimal bandwidth allocation (OBA) method. The bandwidth allocation problem can be formulated as a generic constrained optimization problem:

$$
\text { minimize : } \quad J\left(h_{i}\right)=\sum_{i=1}^{N} J_{i}\left(h_{i}\right),
$$

subject to : $\quad 0 \leq h_{i} \leq \bar{\tau}_{i}$,

$$
b_{1}+b_{2}+\cdots+b_{i}+\frac{\bar{c}_{i}}{h_{i}} \leq i\left(2^{1 / i}-1\right) \text {, }
$$

where $J_{i}\left(h_{i}\right)$ is the QoC of loop $i$ and (22b) and (22c) are the stability constraint and schedulability constraint, respectively. Consider the closed-loop model of loop $i$ :

$$
\dot{x}_{i}(t)=A_{i} x_{i}(t)+B_{i} u_{i}(t), \quad y_{i}(t)=C_{i} x_{i}(t),
$$

where $x_{i}(t)=y_{i}(t)=\omega_{i}(t), u_{i}(t)=i_{i}^{q s}(t), A_{i}=-D_{i} / J_{i}$, $B_{i}=K_{i}^{t} / J_{i}$, and $C_{i}=1$; the subscript $i$ denotes the parameters in loop $i$. Substituting the feedback control law $u_{i}(t)=$ $-K_{i}^{P} x_{i}(k h)$ into (23), the following equation is generated:

$$
\dot{x}_{i}(t)=A_{i} x(t)+M_{i} x_{i}(k h),
$$

where $M_{i}=B_{i} K_{i}^{P}$. The state transmission error is defined as the error in the arrived interval of two contiguous control law packages:

$$
d_{i}(t)=x_{i}(t)-x_{i}\left(t_{k}\right),
$$

with the dynamics of

$$
\begin{aligned}
\dot{d}_{i}(t)=\dot{x}_{i}(t) & =A_{i} x_{i}(t)-M_{i} x_{i}(k h) \\
& =A_{i}\left(d_{i}(t)+x_{i}(k h)\right)-M_{i} x_{i}(k h) \\
& =A_{i} d_{i}(t)+\left(A_{i}-M_{i}\right) x_{i}(k h) .
\end{aligned}
$$

By solving the first-order linear differential equation (26), the Euclidean norm of the ratio between transmitted error and transmitted data can be obtained:

$$
\left\|\frac{d_{i}(t)}{x_{i}(k h)}\right\|=\frac{\left(A_{i}-M_{i}\right)}{A_{i}}\left(1-e^{A_{i} t}\right) .
$$




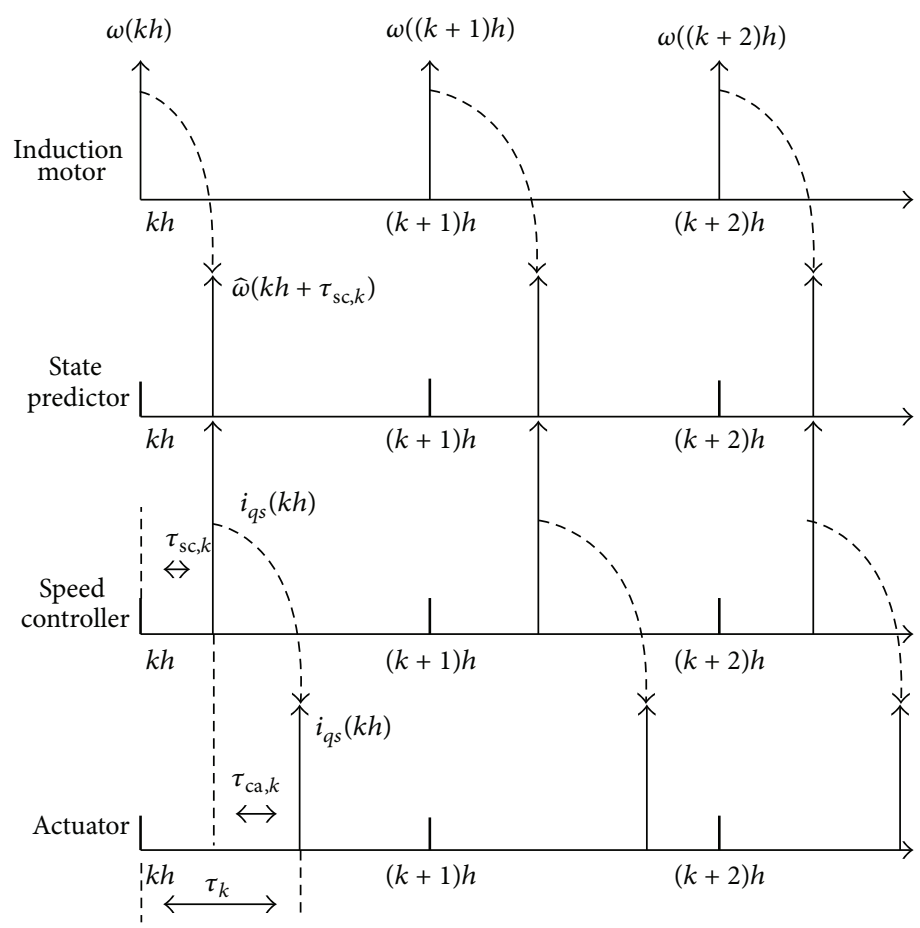

FIGURE 6: Timing diagram of the signals in the NMCS.

Therefore, the performance cost function is defined as

$$
J_{i}\left(h_{i}\right)=\frac{\left(A_{i}-M_{i}\right)}{A_{i}}\left(1-e^{A_{i} h_{i}}\right) .
$$

For $A_{i}<0, J_{i}\left(h_{i}\right)$ is a monotonically increasing function, resulting in the maximization of the QoC which can be formulated as maximizing (28) with constraints.

5.2. Optimal Sampling Period Assignment. In priority-driven network protocols, the control loop with higher data transmission priority has short time delay and lower packet dropouts' rate. In the proposed scheduling method, the higher priority is dynamically assigned to the control loop that more urgently needs to send the message. The key issue of the online priority modification (OPM) method is to assign priorities as a function of the errors obtained from the remote controlled plants. The control loop with larger errors would be assigned with the higher priority. The criterion of assigning priorities is the absolute value of the feedback speed error at each sampling instant:

$$
J_{i}^{\prime}(k)=\left|e_{i}(k)\right|
$$

where $e_{i}(k)=\omega_{i}^{*}(k)-\omega_{i}(k)$, with $\omega_{i}^{*}(k)$ being the reference speed of loop $i$ at the $k$ th sampling instant. Since $J_{i}^{\prime}$ varies over time, a threshold $\delta$ is introduced to reduce the unnecessary priorities switching caused by small variations of QoC. The rules of the OPM method are listed as follows.

(1) If $\max \left\{J_{i}^{\prime}\right\}-\min \left\{J_{i}^{\prime}\right\} \leq \delta$ holds for $i=1, \ldots, N$, then keep the current priorities order.
(2) If $\left|J_{i}^{\prime}(k)-J_{i}^{\prime}(k-1)\right| \leq \delta$ holds for $i=1, \ldots, N$, then keep the current priorities order.

(3) If $\left|J_{i}^{\prime}(k)-J_{j}^{\prime}(k)\right| \leq \delta$ holds, then keep the current priorities order for loop $i$ and loop $j$.

(4) If $J_{i}^{\prime}(k)-J_{j}^{\prime}(k)>\delta$ holds, then $p_{i}(k)>p_{j}(k)$.

5.3. Adjacent Cross Coupling Control. The adjacent cross coupling control structure is proposed to improve the motion coordination performance of multiple induction motors. In the given NMCS including $N$ induction motors with different parameters and load torques, besides $e_{i}(t) \rightarrow 0$ being desired, it is also aimed to regulate the output speed of the motors to satisfy

$$
e_{1}(t)=\cdots=e_{i}(t)=\cdots=e_{N}(t),
$$

which is the requirement on motion coordination operation. Equation (30) can be rewritten as $N$ equations equivalently:

$$
e_{1}(t)=e_{2}(t), \ldots, e_{i}(t)=e_{i+1}(t), \ldots, e_{N}(t)=e_{1}(t) .
$$

Define speed synchronization errors of all adjacent pairs of motors in the following way:

$$
\begin{gathered}
\varepsilon_{1}(t)=e_{1}(t)-e_{2}(t), \\
\vdots \\
\varepsilon_{i}(t)=e_{i}(t)-e_{i+1}(t), \\
\vdots \\
\varepsilon_{n}(t)=e_{n}(t)-e_{1}(t) .
\end{gathered}
$$




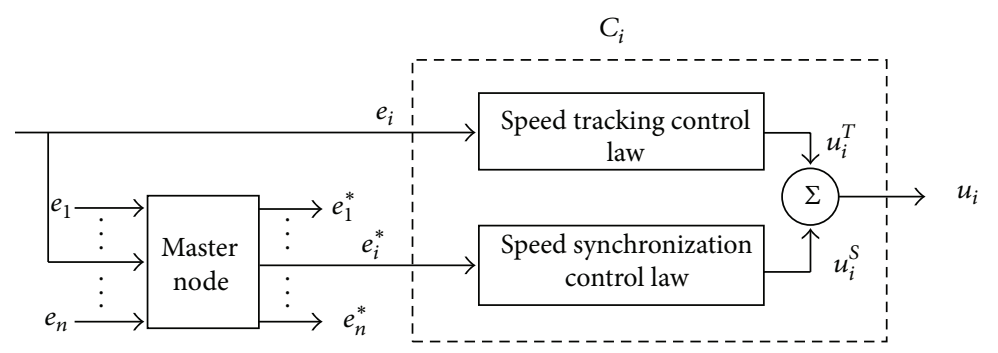

FIgURE 7: Motion coordination operation.

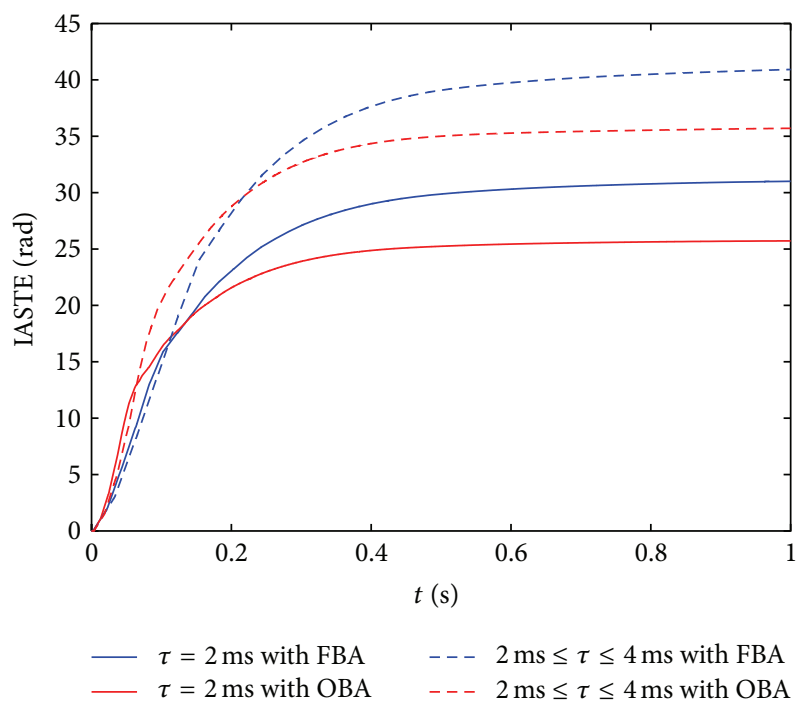

Figure 8: Performance comparison of the FBA and OBA.

If $\varepsilon_{i}(t)=0$ holds for $i=1, \ldots, N$, then (32) holds. The related error variables of motor $i$ are $\varepsilon_{i-1}(t)$ and $\varepsilon_{i}(t)$. Consequently, a new notion named as speed coupled error is introduced:

$$
\begin{gathered}
e_{1}^{*}(t)=\varepsilon_{1}(t)-\varepsilon_{n}(t), \\
\vdots \\
e_{i}^{*}(t)=\varepsilon_{i}(t)-\varepsilon_{i-1}(t), \\
\vdots \\
e_{n}^{*}(t)=\varepsilon_{n}(t)-\varepsilon_{n-1}(t) .
\end{gathered}
$$

If $e_{i}^{*}(t)=0$ holds for $i=1, \ldots, N$, then the speed of the $N$ motors is synchronized.

Motivated by the above analysis, the designed speed controller includes a speed tracking controller and a speed synchronization controller. For the control loop $i, e_{i}^{*}(t)$ is calculated in the scheduler and is sent to the controller $C_{i}$. The speed tracking law and speed synchronization law are calculated in $C_{i}$, which are shown in Figure 7 . The readers can refer to [33] for the adjacent cross coupling control in more detail.

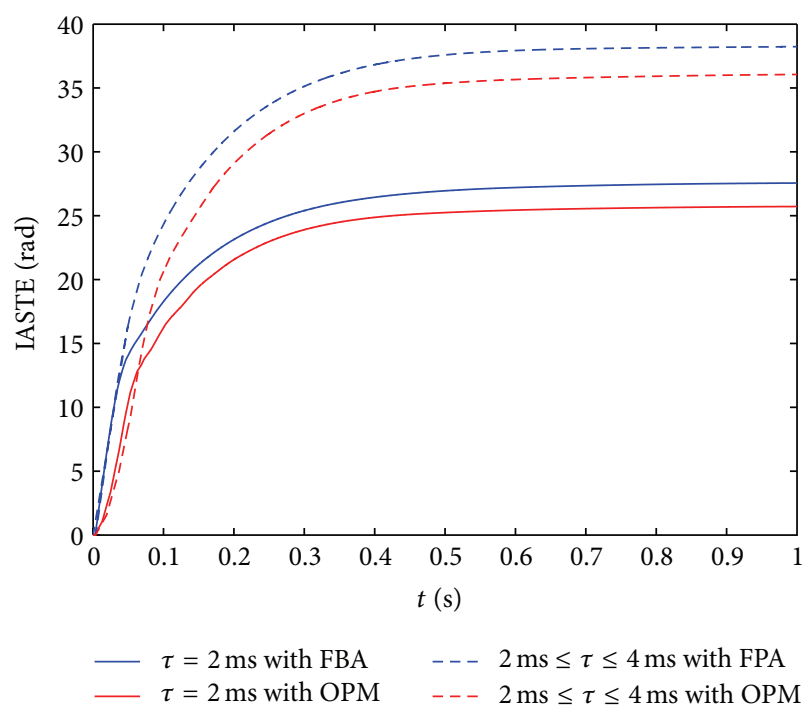

FIGURE 9: Performance comparison of the FPA and OPM.

5.4. Scheduling and Control Codesign. The procedure of the scheduling and control codesign is shown in Algorithm 1. The performance of the proposed optimal bandwidth scheduling and online priority modification schemes are evaluated by the integral of absolute speed tracking errors (IASTE):

$$
\operatorname{IASTE}=\sum_{i=1}^{N} \int_{0}^{\infty}\left|e_{i}(t)\right| d t .
$$

Similarly, the performance of the proposed adjacent cross coupling control structure is evaluated by the integral of the absolute speed synchronization errors (IASSE):

$$
\operatorname{IASSE}=\sum_{i=1}^{N} \int_{0}^{\infty}\left|\varepsilon_{i}(t)\right| d t
$$

\section{Simulation Results}

To verify the proposed codesign procedure and demonstrate its effectiveness, simulation studies are carried out for a NMCS with 4 control loops using the TrueTime toolbox on MATLAB/Simulink. The network type is CSMA/AMP (CAN), the data rate is $80 \mathrm{Kbits} / \mathrm{s}$, and the minimum frame size is 32 bits. The reference speed of the induction motors is 


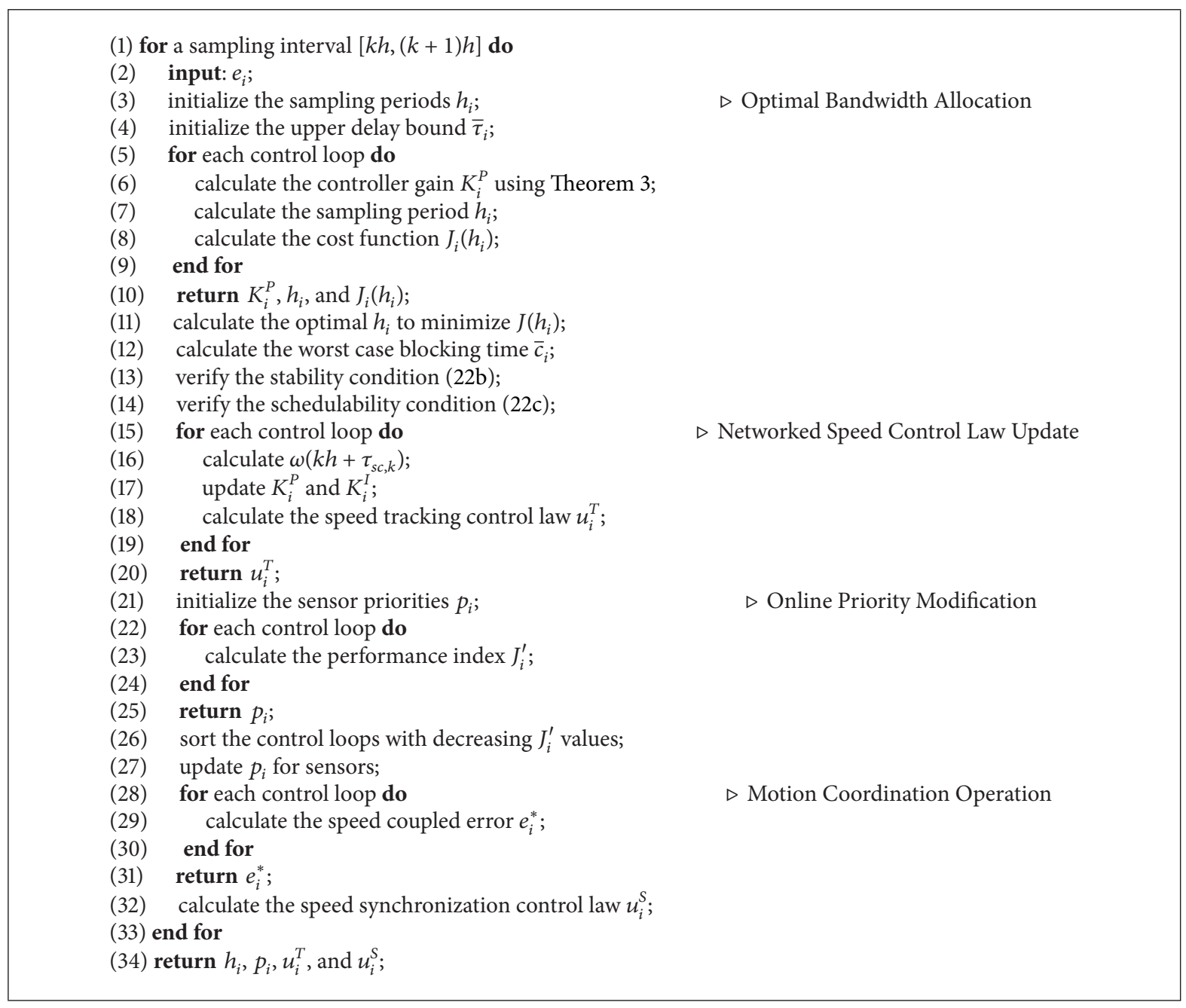

Algorithm 1: Scheduling and control codesign.

set as an identical value of $\omega^{*}=100 \mathrm{rad} / \mathrm{s}$. Parameters of the 4 motors in simulation are listed in Table 2.

Simulation results are done under two typical QoS conditions: (1) short and constant transmission time ( $\tau_{i}=2 \mathrm{~ms}$ ); (2) long and time-varying transmission time ( $\left.2 \mathrm{~ms} \leq \tau_{i} \leq 4 \mathrm{~ms}\right)$. Substituting $\bar{\tau}$ into Theorem 3 , the obtained upper allowed feedback gains of the four control loops are shown in Table 3.

The simulation studies are conducted in 5 different cases, which are

(1) the comparison of the proposed OBA scheme with the fixed bandwidth allocation (FBA) scheme;

(2) the comparison of the OPM scheme with the fixed priority assignment (FPA) scheme;

(3) the comparison of the fuzzy logic speed controller with the memoryless state feedback speed controller;

(4) performance evaluation of the state predictor in time delay compensation;

(5) performance evaluation of the adjacent cross coupling control structure in motion coordination.
The simulation results are illustrated in the following.

Case 1. In the FBA scheme, the sampling period of each loop is selected as identical, while satisfying both of the stability constraint and the schedulability constraint. Under the two QoS conditions, the sampling period is selected as $h_{i}=0.02 \mathrm{~s}$ and $h_{i}=0.03 \mathrm{~s}$, respectively. In the OBA scheme, the optimization problem can be solved using the MATLAB function fmincon, and the optimized sampling period of all the loops is listed in Table 4 . The simulation results are demonstrated in Figure 8, where the IASTE are reduced under both of the test conditions using the proposed OBA scheme.

Case 2. The comparison of the OPM scheme with the FPA scheme is shown in Figure 9. In the FPA scheme, the initial priority of each loop is identical to its index $(1,2, \ldots, N)$. The simulation results show that the IASTE with OPM are less than those with FPA under both test conditions, which showed the effectiveness of the proposed scheduling method. 
TABle 2: Parameters of induction motors.

\begin{tabular}{lcccc}
\hline Parameters & Motor 1 & Motor 2 & Motor 3 & Motor 4 \\
\hline$R_{s} / \Omega$ & 6.700 & 5.460 & 3.670 & 8.000 \\
$R_{r} / \Omega$ & 5.500 & 4.450 & 2.100 & 3.600 \\
$L_{s} / \mathrm{H}$ & 0.475 & 0.492 & 0.245 & 0.470 \\
$L_{r} / \mathrm{H}$ & 0.475 & 0.492 & 0.245 & 0.470 \\
$M / \mathrm{H}$ & 0.450 & 0.475 & 0.224 & 0.450 \\
$J /\left(\mathrm{kgm}^{2}\right)$ & 0.015 & 0.015 & 0.016 & 0.015 \\
$\psi_{r}^{*} / \mathrm{Wb}$ & 1.000 & 1.000 & 1.000 & 1.000 \\
\hline
\end{tabular}

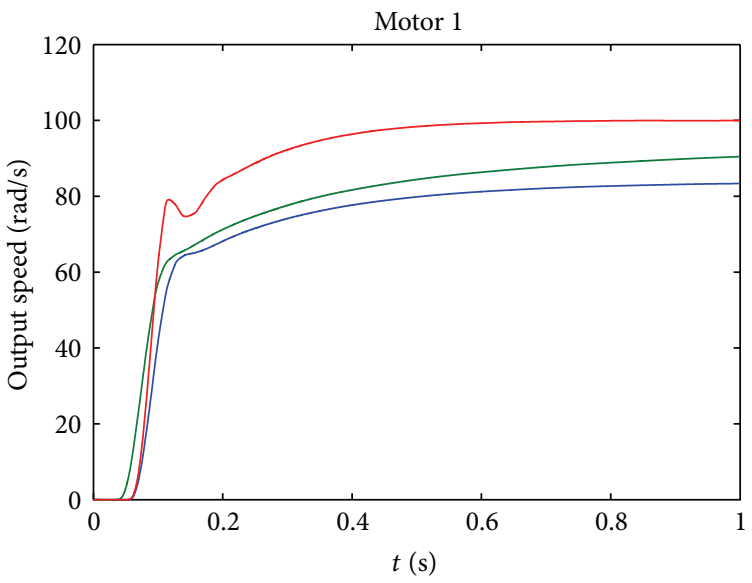

(a)

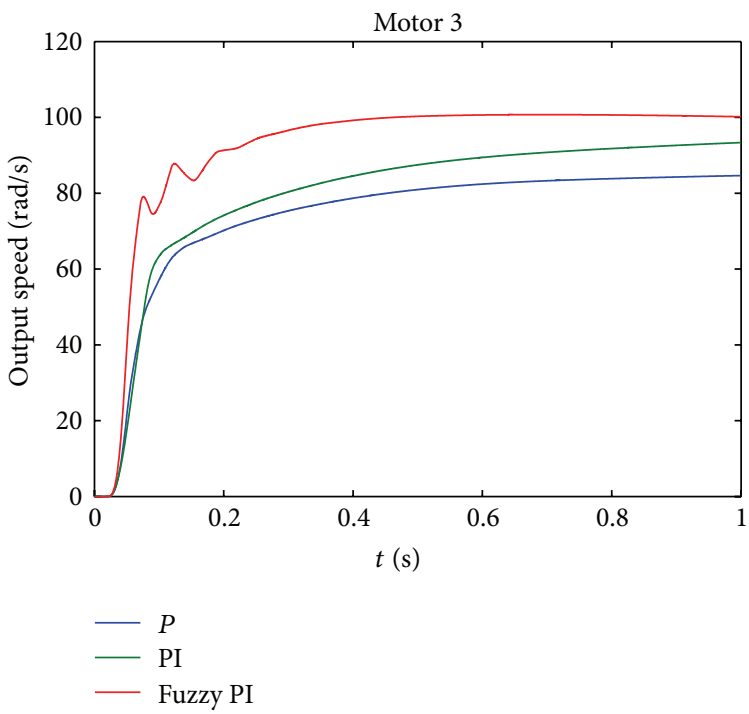

(c)

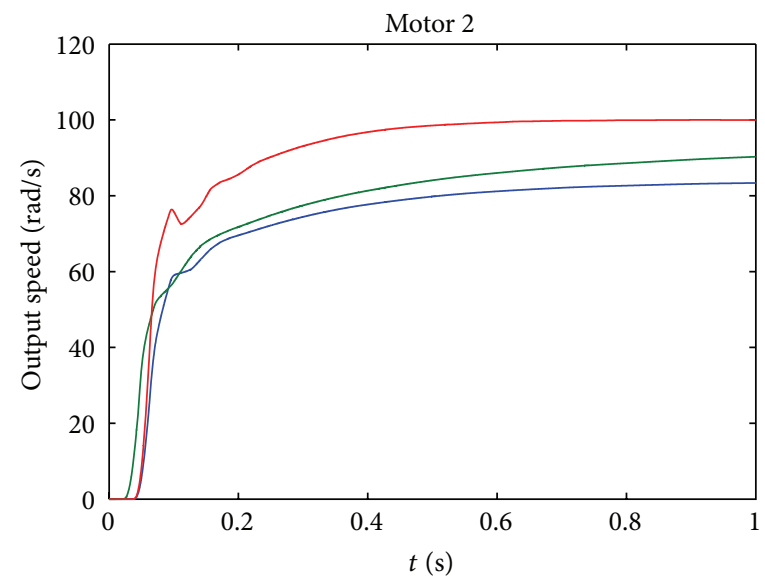

(b)

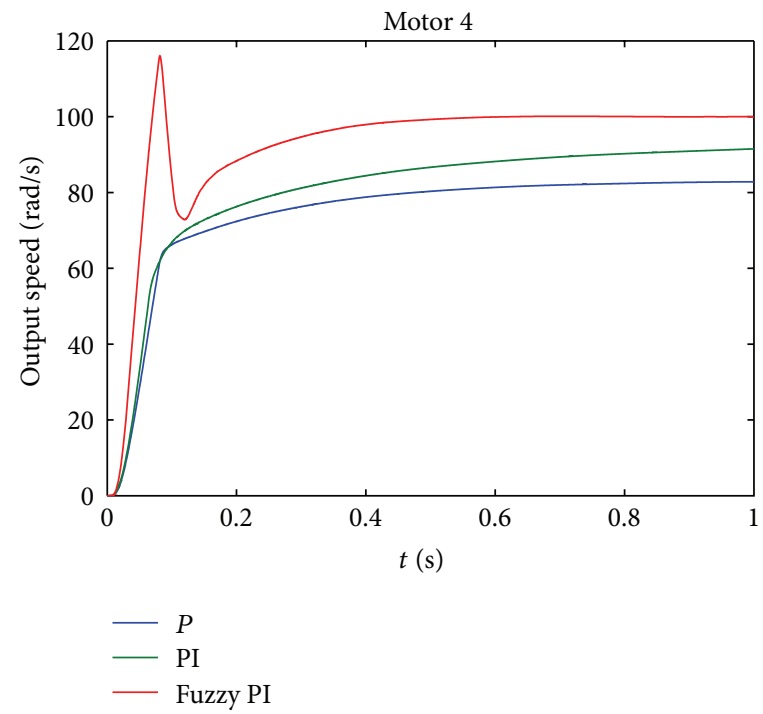

(d)

FIGURE 10: Comparison of networked controllers in condition 2.

TABLE 3: Upper limits of the feedback gains.

\begin{tabular}{lllll}
\hline QoS condition & $\bar{K}_{1}^{P}$ & $\bar{K}_{2}^{P}$ & $\bar{K}_{3}^{P}$ & $\bar{K}_{4}^{P}$ \\
\hline Condition 1 & 4.0 & 4.0 & 2.5 & 4.0 \\
Condition 2 & 2.0 & 2.0 & 1.5 & 2.0 \\
\hline
\end{tabular}

TABLE 4: The optimal sampling period of each loop.

\begin{tabular}{lcccc}
\hline QoS condition & $h_{1}$ & $h_{2}$ & $h_{3}$ & $h_{4}$ \\
\hline Condition 1 & 0.010 & 0.010 & 0.011 & 0.011 \\
Condition 2 & 0.020 & 0.020 & 0.022 & 0.022 \\
\hline
\end{tabular}




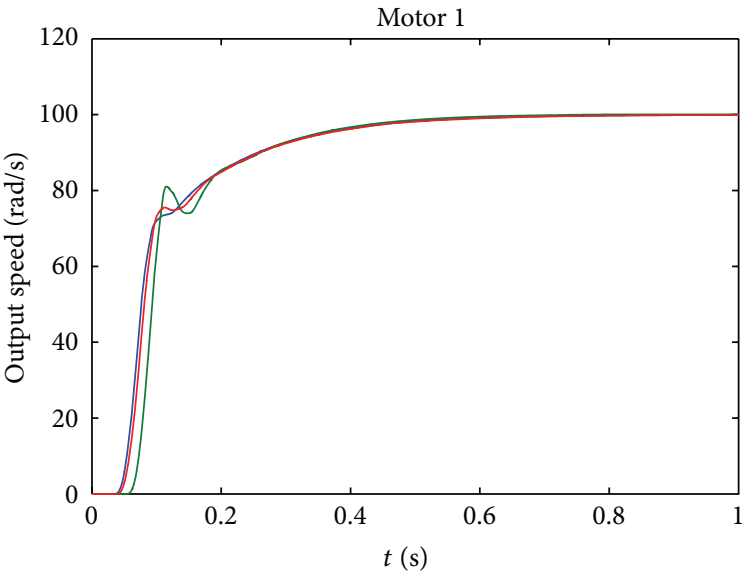

(a)

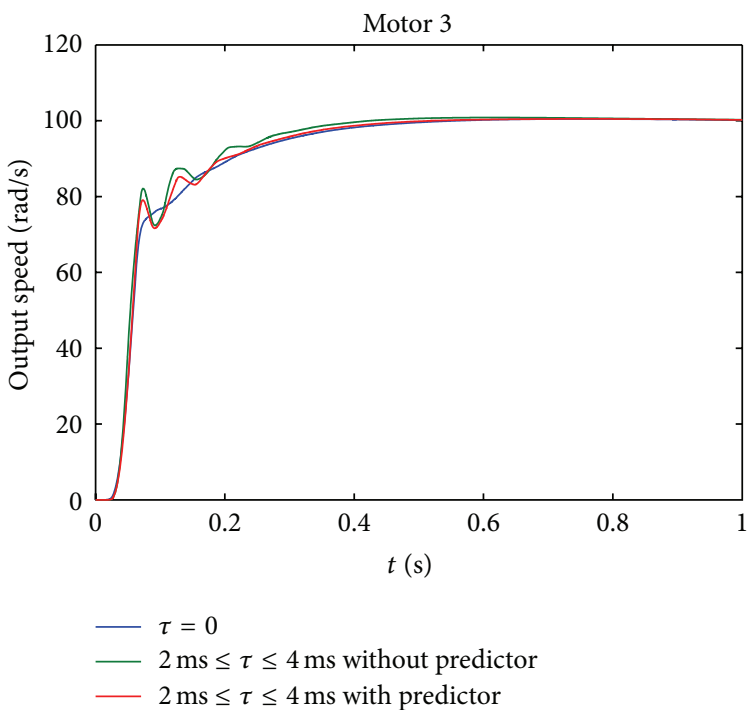

(c)

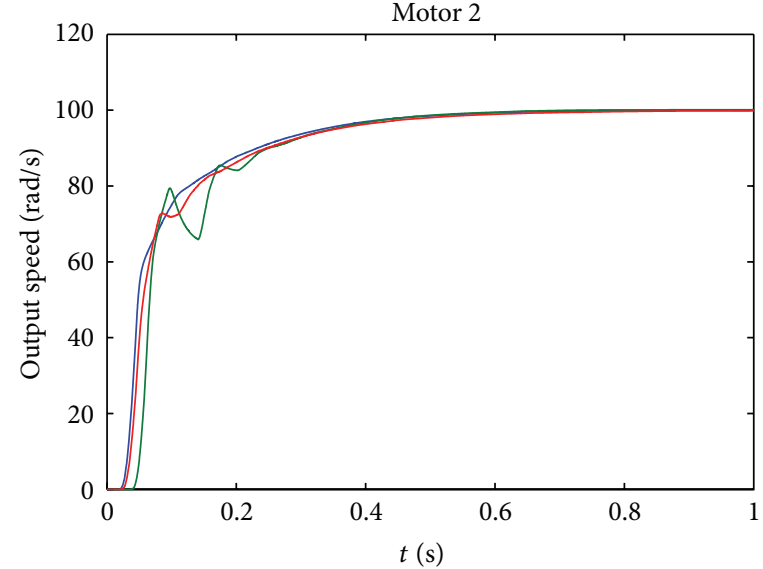

(b)

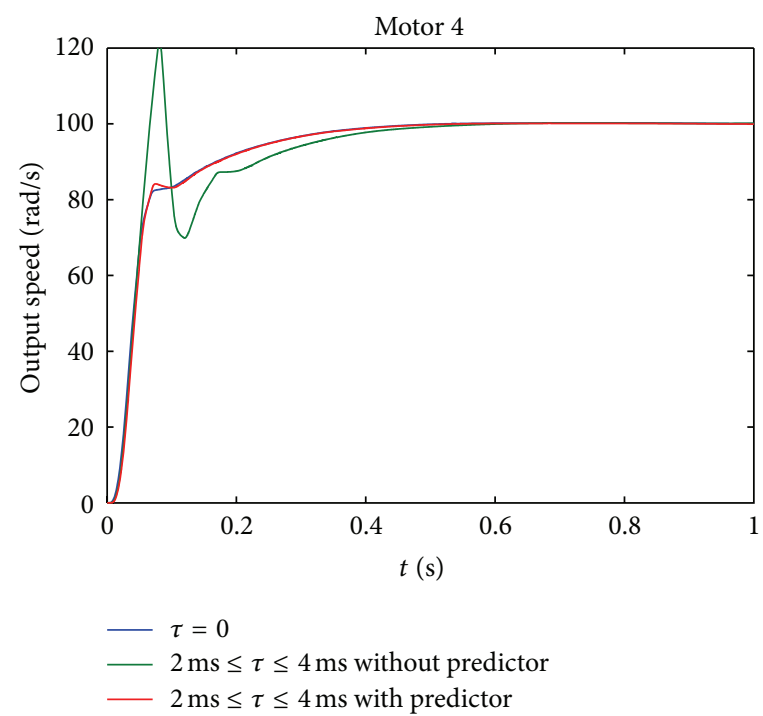

(d)

FIgURE 11: Performance evaluation of the state predictor in condition 2.

For the OPM method being applied on the application layer, modification on the network MAC protocol is not required.

Case 3. The comparison of different networked controllers is presented in Figure 10. The system performance using a $P$ controller has the slowest response and largest steady error. This is reasonable since it uses the least information about the system. By employing a PI controller, the steady error of the NMCS is improved, but the dynamic response still cannot meet the requirement. However, using the proposed fuzzy logic tuning PI controller, the dynamic response is fast and the steady error is much smaller than the above two controllers, for the fuzzy PI controller being able to tune its gains adaptively according to the output and QoS.

Case 4. The performance evaluation of the state predictor under the appointed two conditions is illustrated in Figure 11. When the NMCS suffers no time delay, the speed tracking performance is the best. When $\tau$ is induced and without delay compensation, the system performance deteriorated. With the state predictor applied, the speed tracking performance is improved, especially on the oscillation.

Case 5. The performance evaluation of the adjacent cross coupling control structure is given in Figure 12. The proposed method reduces the IASSE under both of the test conditions, which shows that the proposed cross coupling control strategy is effective to synchronize the output speed of multiple motors.

\section{Conclusions}

In this paper, an integrated feedback scheduling strategy is proposed for motion coordination operation of multiple induction motors via a shared control network, and its codesign with a networked speed controller was developed. The scheduling strategy includes the optimal bandwidth allocation scheme, online priority modification scheme, 


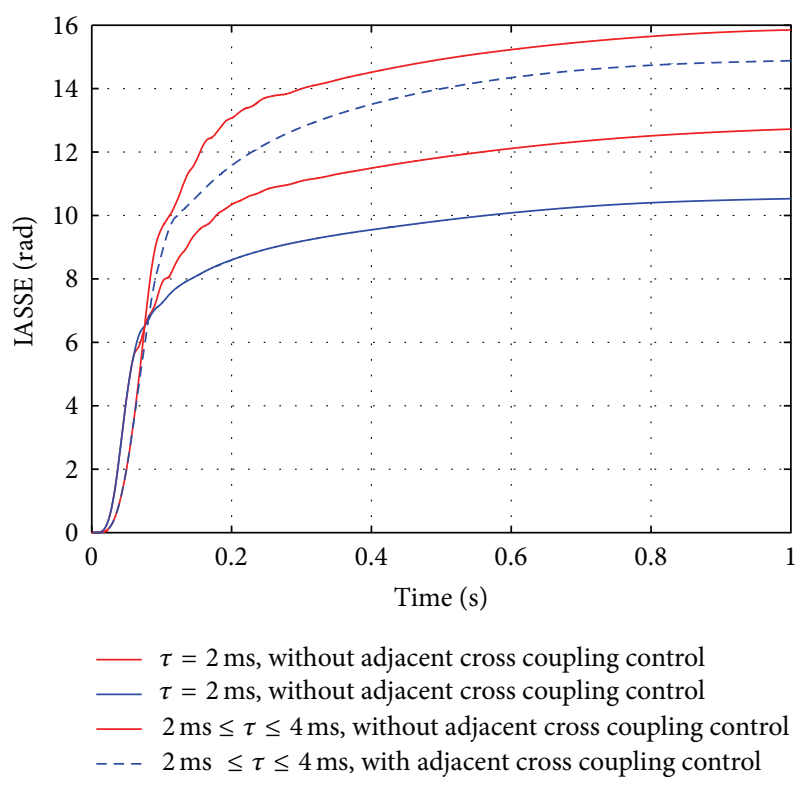

FIgURE 12: Performance evaluation of the motion coordination operation.

and adjacent cross coupling control scheme. The optimal bandwidth allocation scheme minimized the transmission errors, satisfying the stability constraint and the schedulability constraint. The online priority modification scheme decided the data transmission order by sorting the realtime speed feedback errors; therefore the control loops can send their data packet according to their urgency level. The adjacent cross coupling control scheme improved the speed synchronization performance in a simplified control structure. The upper limit of the gain of the static feedback networked speed controller is calculated employing the Lyapunov theorem. Furthermore, the closed-loop control performance was improved by online tuning of the gains, together with a state predictor in the feedback channel. Simulation results were conducted in several cases and demonstrated the effectiveness of the codesign methodology under constant delay and time-variable delay, respectively.

\section{Appendix}

Proof. Select a Lyapunov function as

$$
V(x(t))=V_{1}+V_{2}+V_{3}
$$

with

$$
\begin{gathered}
V_{1} \triangleq x^{T}(t) P x(t) \\
V_{2} \triangleq \sum_{i=1}^{N}\left\{\int_{-\tau_{i}}^{0} \int_{t+\beta}^{t} \dot{x}^{T}(\alpha) Z \dot{x}(\alpha) d \alpha d \beta\right\}, \\
V_{3} \triangleq \sum_{i=1}^{N} \int_{t-\tau_{i}}^{t} x^{T}(\alpha) Q x(\alpha) d \alpha .
\end{gathered}
$$

Taking into account the Newton-Leibniz formula

$$
\begin{aligned}
x\left(t-\tau_{i}\right) & =x(t)-\int_{t-\tau_{i}}^{t} \dot{x}(\sigma) d \sigma \\
& =x(t)-\int_{t-\tau_{i}}^{t}\left[F x(\sigma)+\sum_{i=1}^{N}\left\{F_{i} x\left(\sigma-\tau_{i}\right)\right\}\right] d \sigma,
\end{aligned}
$$

the NMCS model (11) can be written as

$$
\dot{x}(t)=\left(F+\sum_{i=1}^{N} F_{i}\right) x(t)-\sum_{i=1}^{N}\left\{F_{i} \int_{t-\tau_{i}}^{t} \dot{x}(\alpha) d \alpha\right\} .
$$

Therefore, the derivative of $V_{1}$ is expressed as

$$
\begin{aligned}
\dot{V}_{1}= & 2 x^{T}(t)\left[P\left(F+\sum_{i=1}^{N} F_{i}\right)\right] x(t) \\
& -2 \sum_{i=1}^{N}\left\{x^{T}(t) P F_{i} \int_{t-\tau_{i}}^{t} \dot{x}(\alpha) d \alpha\right\} .
\end{aligned}
$$

For all $\alpha \in[t-\tau, t]$, the terms in Lemma 1 are defined as $a(\alpha) \triangleq x(t), b(\alpha) \triangleq \dot{x}(\alpha)$, and $W \triangleq P F_{i}$. Using Lemma 1 and $\left[\begin{array}{cc}X_{i} & Y_{i} \\ Y_{i}^{T} & Z_{i}\end{array}\right] \geq 0(i=1, \ldots, N)$, the following inequalities hold:

$$
\begin{aligned}
\dot{V}_{1} \leq & 2 x^{T}(t)\left[P\left(F+\sum_{i=1}^{N} F_{i}\right)\right] x(t)+\sum_{i=1}^{N} \tau_{i} x^{T}(t) X_{i} x(t) \\
& +2 \sum_{i=1}^{N}\left\{x^{T}(t)\left(Y_{i}-P F_{i}\right) \int_{t-\tau_{i}}^{t} \dot{x}(\alpha) d \alpha\right\} \\
& +\sum_{i=1}^{N}\left\{\int_{t-\tau_{i}}^{t} \dot{x}^{T}(\alpha) Z_{i} \dot{x}(\alpha) d \alpha\right\}
\end{aligned}
$$$$
\leq x^{T}(t)\left[F^{T} P+P F+\sum_{i=1}^{N}\left\{\bar{\tau} X_{i}+Y_{i}+Y_{i}^{T}\right\}\right] x(t)
$$$$
+2 \sum_{i=1}^{N}\left\{x^{T}(t)\left(P F_{i}-Y_{i}\right) x\left(t-\tau_{i}\right)\right\}
$$$$
+\sum_{i=1}^{N}\left\{\int_{t-\tau_{i}}^{t} \dot{x}^{T}(\alpha) Z_{i} \dot{x}(\alpha) d \alpha\right\}
$$ 


$$
\begin{gathered}
\dot{V}_{2}=\sum_{i=1}^{N}\left\{\tau_{i}\left[F x(t)+\sum_{i=1}^{N}\left\{F_{i} x\left(t-\tau_{i}\right)\right\}\right]_{i}^{T}\right. \\
\left.\times\left[F x(t)+\sum_{i=1}^{N}\left\{F_{i} x\left(t-\tau_{i}\right)\right\}\right]\right\} \\
-\sum_{i=1}^{N}\left\{\int_{t-\tau}^{t} \dot{x}^{T}(\alpha) Z \dot{x}(\alpha) d \alpha\right\}, \\
\dot{V}_{3}=\sum_{i=1}^{N}\left\{x^{T}(t) Q_{i} x(t)-x^{T}\left(t-\tau_{i}\right) Q_{i} x\left(t-\tau_{i}\right)\right\} .
\end{gathered}
$$

Therefore, the derivative of the Lyapunov function $V$ has the following characteristics:

$$
\dot{V}=\dot{V}_{1}+\dot{V}_{2}+\dot{V}_{3} \leq \bar{x}^{T}\left[\begin{array}{ll}
X_{11} & X_{12} \\
X_{12}^{T} & X_{22}
\end{array}\right]^{T} \bar{x},
$$

where

$$
\begin{aligned}
& \bar{x}=\left[\begin{array}{lllll}
x^{T}(t) & x^{T}\left(t-\tau_{1}\right) & \cdots & x^{T}\left(t-\tau_{N}\right)
\end{array}\right]^{T}, \\
& X_{11} \triangleq F^{T} P+P F \\
& +\sum_{i=1}^{N}\left\{Y_{i}+Y_{i}^{T}+\bar{\tau} X_{i}+Q_{i}+\bar{\tau} F^{T} Z_{i} F\right\}, \\
& X_{12} \triangleq\left[F_{1}-Y_{1}+\bar{\tau} \sum_{i=1}^{N} F^{T} Z_{i} F_{1} \cdots\right. \\
& \left.F_{N}-Y_{N}+\bar{\tau} \sum_{i=1}^{N} F^{T} Z_{i} F_{N}\right], \\
& X_{22} \triangleq-\operatorname{diag}\left[\begin{array}{lll}
Q_{1} & \cdots & Q_{N}
\end{array}\right] \\
& +\left[\begin{array}{ccc}
\sum_{i=1}^{N}\left\{\bar{\tau} F_{1}^{T} Z_{i} F_{1}\right\} & \cdots & \sum_{i=1}^{N}\left\{\bar{\tau} F_{1}^{T} Z_{i} F_{N}\right\} \\
\vdots & \ddots & \vdots \\
\sum_{i=1}^{N}\left\{\bar{\tau} F_{N}^{T} Z_{i} F_{1}\right\} & \cdots & \sum_{i=1}^{N}\left\{\bar{\tau} F_{N}^{T} Z_{i} F_{N}\right\}
\end{array}\right] .
\end{aligned}
$$

Therefore, if

$$
\left[\begin{array}{ll}
X_{11} & X_{12} \\
X_{12}^{T} & X_{22}
\end{array}\right]<0
$$

then system (11) is asymptotically stable according to the Lyapunov theorem. The inequality (A.9) can be rewritten as

$$
\mathscr{F}^{T} \mathscr{Z} \Gamma^{-1} \mathscr{Z}^{T} \mathscr{F}+\left[\begin{array}{cc}
Y_{11} & Y_{12} \\
Y_{12}^{T} & Y_{22}
\end{array}\right]<0,
$$

where

$$
\begin{gathered}
Y_{11} \triangleq F^{T} P+P F+\sum_{i=1}^{N}\left\{Y_{i}+Y_{i}^{T}+\bar{\tau} X_{i}+Q_{i}\right\}, \\
Y_{12} \triangleq\left[P F_{1}-Y_{1} \cdots P F_{N}-Y_{N}\right], \\
Y_{22} \triangleq-\operatorname{diag}\left\{Q_{1}, \ldots, Q_{N}\right\} .
\end{gathered}
$$

Define the matrices as

$$
\mathscr{A} \triangleq \mathscr{F}^{T} \mathscr{Z}, \quad \mathscr{P} \triangleq \Gamma^{-1}, \quad \mathbb{Q} \triangleq\left[\begin{array}{ll}
Y_{11} & Y_{12} \\
Y_{12}^{T} & Y_{22}
\end{array}\right] .
$$

Using Lemma 2, (15) is an expression of the first inequality of (14). Considering

$$
\mathscr{A} \mathscr{P} \mathscr{A}^{T}+\mathscr{Q}=\left[\begin{array}{ll}
X_{11} & X_{12} \\
X_{12}^{T} & X_{22}
\end{array}\right],
$$

it is concluded that the system (11) is asymptotically stable if (15) holds. This completes the proof.

\section{Conflict of Interests}

The authors declare that there is no conflict of interests regarding the publication of this paper.

\section{Acknowledgment}

This work was supported by the National Natural Science Foundation of China under the Grant reference 61174068.

\section{References}

[1] Y. Cao, D. Stuart, W. Ren, and Z. Meng, "Distributed containment control for multiple autonomous vehicles with doubleintegrator dynamics: algorithms and experiments," IEEE Transactions on Control Systems Technology, vol. 19, no. 4, pp. 929938, 2011.

[2] P. Millan, L. Orihuela, G. Bejarano, C. Vivas, T. Alamo, and F. R. Rubio, "Design and application of suboptimal mixed $H_{2} / H_{\infty}$ controllers for networked control systems," IEEE Transactions on Control Systems Technology, vol. 20, no. 4, pp. 1057-1065, 2012.

[3] M. Schwager, B. J. Julian, M. Angermann, and D. Rus, "Eyes in the sky: decentralized control for the deployment of robotic camera networks," Proceedings of the IEEE, vol. 99, no. 9, pp. 1541-1561, 2011.

[4] K. Natori, T. Tsuji, K. Ohnishi, A. Hace, and K. Jezernik, "Timedelay compensation by communication disturbance observer for bilateral teleoperation under time-varying delay," IEEE Transactions on Industrial Electronics, vol. 57, no. 3, pp. 1050 1062, 2010.

[5] H. Wu, L. Lou, C. C. Chen, S. Hirche, and K. Kuhnlenz, "Cloudbased networked visual servo control," IEEE Transactions on Industrial Electronics, vol. 60, no. 2, pp. 554-566, 2013.

[6] J. Ferruz, V. M. Vega, A. Ollero, and V. Blanco, "Reconfigurable control architecture for distributed systems in the HERO autonomous helicopter," IEEE Transactions on Industrial Electronics, vol. 58, no. 12, pp. 5311-5318, 2011. 
[7] J. Tisdale, Z. W. Kim, and J. K. Hedrick, "Autonomous UAV path planning and estimation: an online path planning framework for cooperative search and localization," IEEE Robotics and Automation Magazine, vol. 16, no. 2, pp. 35-42, 2009.

[8] I. Maza, K. Kondak, M. Bernard, and A. Ollero, "Multi-UAV cooperation and control for load transportation and deployment," Journal of Intelligent and Robotic Systems: Theory and Applications, vol. 57, no. 1-4, pp. 417-449, 2010.

[9] J. Marescaux, J. Leroy, M. Gagner et al., "Transatlantic robotassisted telesurgery," Nature, vol. 413, no. 6854, pp. 379-380, 2001.

[10] H. J. Cho, H. Son, D. G. Lee, T. Bhattacharjee, and D. Y. Lee, "Gain-scheduling control of teleoperation systems interacting with soft tissues," IEEE Transactions on Industrial Electronics, vol. 60, no. 3, pp. 946-957, 2013.

[11] S. Li, Y. Sun, and J. Qian, "Research on real time networked control platforn for coal mine and applications," in Proceedings of the International Conference on Information Acquisition (ICIA '07), pp. 427-431, July 2007.

[12] B. K. Kim, W. K. Chung, and I. H. Suh, "Robust synchronizing motion control of twin-servo systems based on network modeling," in Proceedings of the 39th IEEE Confernce on Decision and Control, pp. 1019-1024, December 2000.

[13] H. Li, M.-Y. Chow, and Z. Sun, "EDA-based speed control of a networked DC motor system with time delays and packet losses," IEEE Transactions on Industrial Electronics, vol. 56, no. 5, pp. 1727-1735, 2009.

[14] H. Zhang, Y. Shi, and A. Saadat Mehr, "Robust static output feedback control and remote PID design for networked motor systems," IEEE Transactions on Industrial Electronics, vol. 58, no. 12, pp. 5396-5405, 2011.

[15] K.-Y. Lian, C.-Y. Hung, C.-S. Chiu, and P. Liu, "Induction motor control with friction compensation: an approach of virtual-desired-variable synthesis," IEEE Transactions on Power Electronics, vol. 20, no. 5, pp. 1066-1074, 2005.

[16] C. Y. Hung, P. Liu, and K. Y. Lian, "Fuzzy virtual reference model sensorless tracking control for linear induction motors," IEEE Transactions on Cybernetics, vol. 43, no. 3, pp. 970-981, 2013.

[17] P. Liu, C. Y. Hung, C. S. Chiu, and K. Y. Lian, "Sensorless linear induction motor speed tracking using fuzzy observers," IET Electric Power Applications, vol. 5, no. 4, pp. 325-334, 2011.

[18] L. X. Zhang, H. J. Gao, and O. Kaynak, "Network-induced constraints in networked control systems-a survey," IEEE Transactions on Industrial Informatics, vol. 9, no. 1, pp. 403-416, 2013.

[19] M. C. F. Donkers, W. P. M. H. Heemels, N. van de Wouw, and L. Hetel, "Stability analysis of networked control systems using a switched linear systems approach," IEEE Transactions on Automatic Control, vol. 56, no. 9, pp. 2101-2115, 2011.

[20] D. Wu, J. Wu, S. Chen, and J. Chu, "Stability of networked control systems with polytopic uncertainty and buffer constraint," IEEE Transactions on Automatic Control, vol. 55, no. 5, pp. 12021208, 2010.

[21] D. Zhao, C. Li, and J. Ren, "Fuzzy speed control and stability analysis of a networked induction motor system with time delays and packet dropouts," Nonlinear Analysis: Real World Applications, vol. 12, no. 1, pp. 273-287, 2011.

[22] H. Xu and S. Jagannathan, "Stochastic optimal controller design for uncertain nonlinear networked control system via neuro dynamic programming," IEEE Transactions on Neural Networks and Learning Systems, vol. 24, no. 3, pp. 471-484, 2013.
[23] H. Zhang, Y. Shi, and M. X. Liu, " $H_{\infty}$ step tracking control for networked discrete-time nonlinear systems with integral and predictive actions," IEEE Transactions on Industrial Informatics, vol. 9, no. 1, pp. 337-345, 2013.

[24] H. Benitez-Perez, A. Benitez-Perez, and J. Ortega-Arjona, "Networked control systems design considering scheduling restrictions and local faults," International Journal of Innovative Computing Information and Control, vol. 8, no. 12, pp. 85158526, 2012.

[25] C. L. Liu and J. W. Layland, "Scheduling algorithms for multiprogramming in a hard-real-time environment," Journal of the Association for Computing Machinery, vol. 20, pp. 46-61, 1973.

[26] M. E. M. Ben Gaid, A. Çela, and Y. Hamam, "Optimal integrated control and scheduling of networked control systems with communication constraints: application to a car suspension system," IEEE Transactions on Control Systems Technology, vol. 14, no. 4, pp. 776-787, 2006.

[27] M. S. Branicky, S. M. Phillips, and W. Zhang, "Scheduling and feedback co-design for networked control systems," in Proceedings of the 41st IEEE Conference on Decision and Control, vol. 1-4, pp. 1211-1217, December 2002.

[28] S.-L. Dai, H. Lin, and S. S. Ge, "Scheduling-and-control codesign for a collection of networked control systems with uncertain delays," IEEE Transactions on Control Systems Technology, vol. 18, no. 1, pp. 66-78, 2010.

[29] B. K. Bose, Modern Power Electronics and AC Drives, Prentice Hall, New Jersey, NJ, USA, 2002.

[30] Z. Yan and V. Utkin, "Sliding mode observers for electric machines-an overview," in Proceedings of the 2002 28th Annual Conference of the IEEE Industrial Electronics Society, pp. 18421847, November 2002.

[31] P. Park, Y. S. Moon, and W. H. Kwon, "A delay-dependent robust stability criterion for uncertain time-delay systems," in Proceedings of the 1998 American Control Conference, pp. 19631964, 1998.

[32] S. Boyd, L. El Ghaoui, E. Feron, and V. Balakrishnan, Linear Matrix Inequalities in System and Control Theory, vol. 15 of SIAM Studies in Applied Mathematics, SIAM, Philadelphia, Pa, USA, 1994.

[33] D. Z. Zhao, C. W. Li, and J. Ren, "Speed synchronisation of multiple induction motors with adjacent cross-coupling control," IET Control Theory \& Applications, vol. 4, no. 1, pp. 119$128,2010$. 


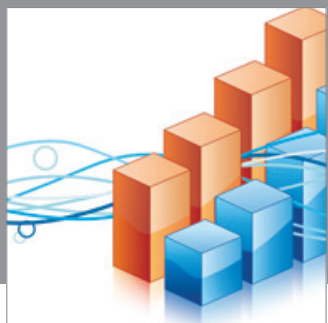

Advances in

Operations Research

mansans

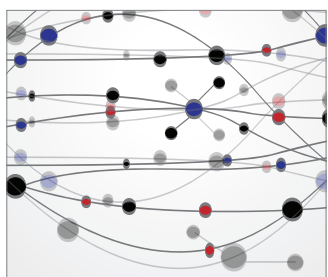

The Scientific World Journal
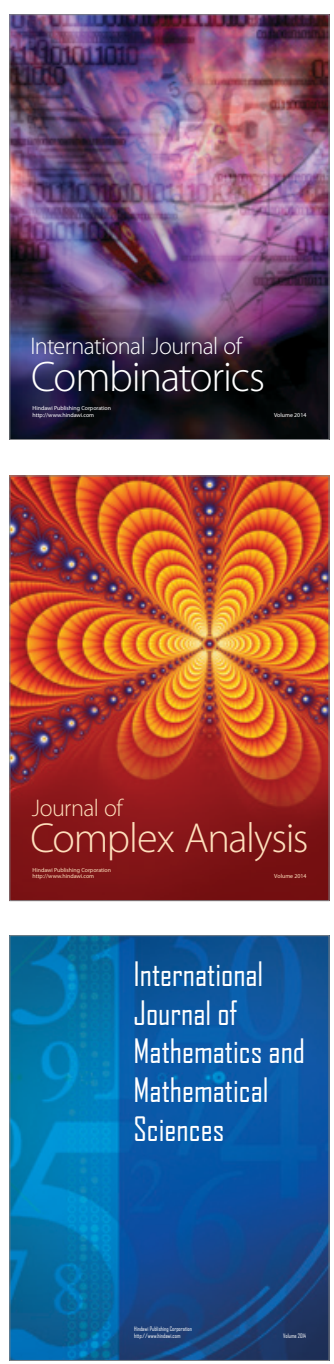
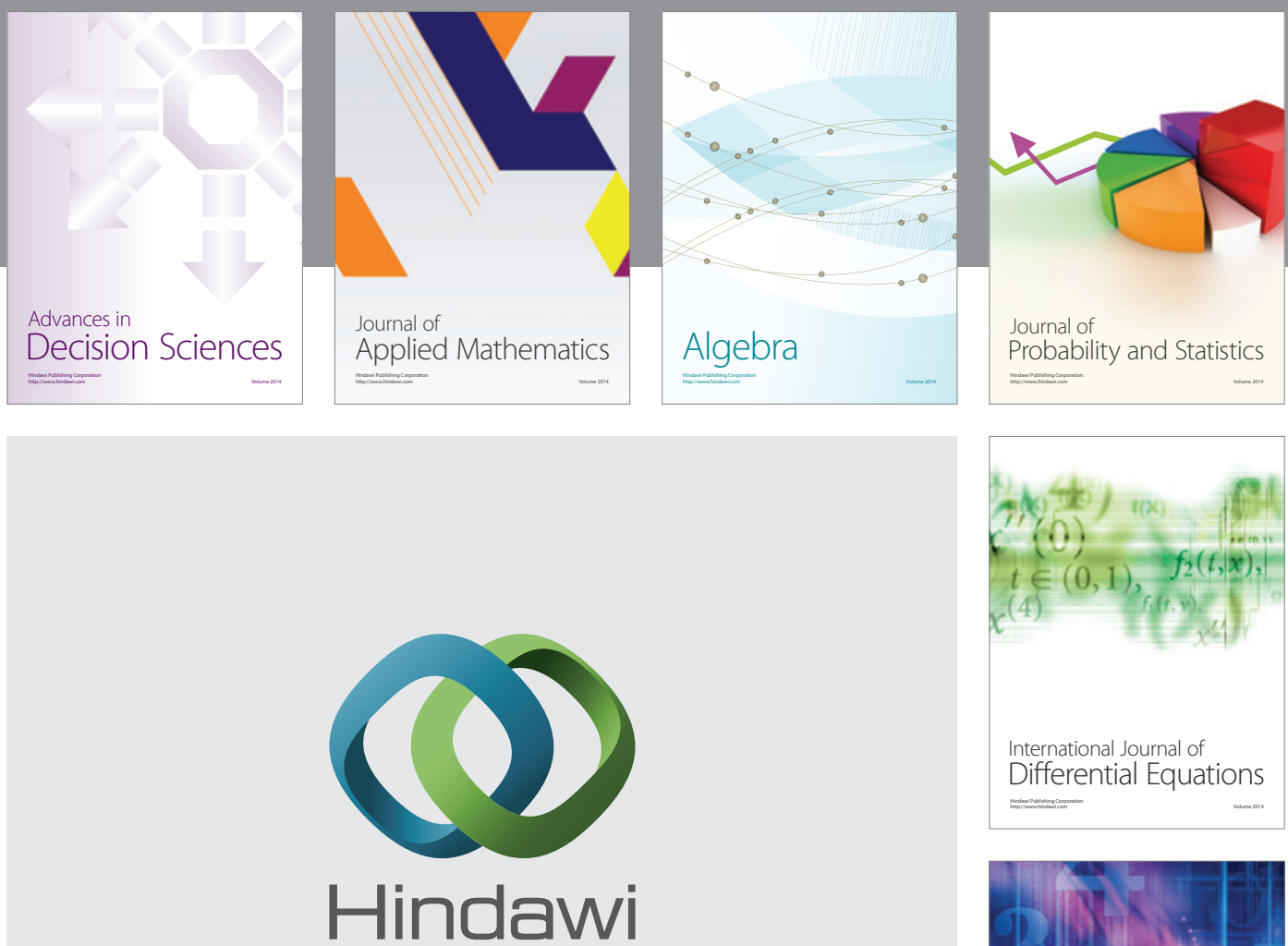

Submit your manuscripts at http://www.hindawi.com
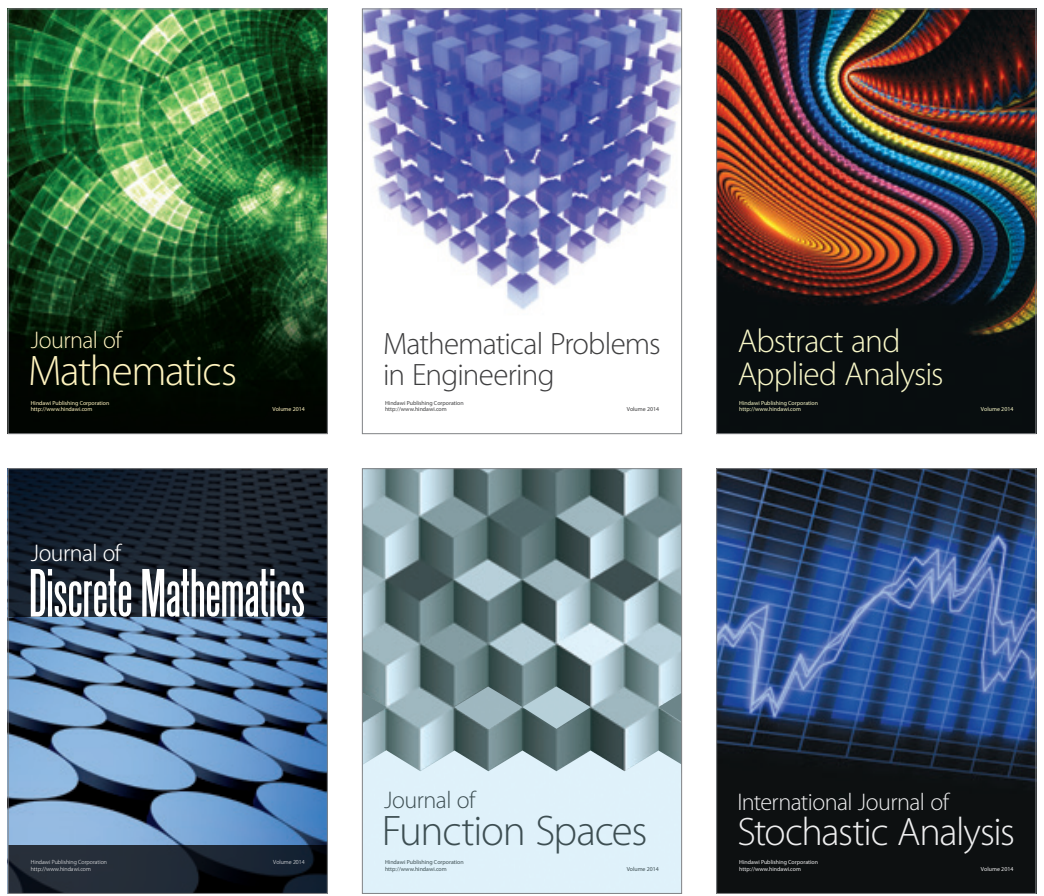

Journal of

Function Spaces

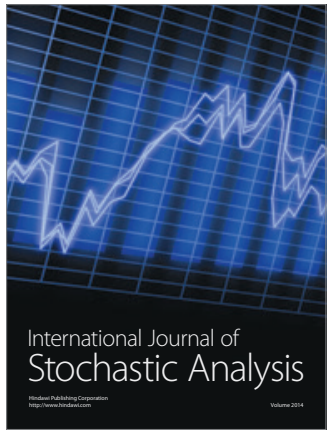

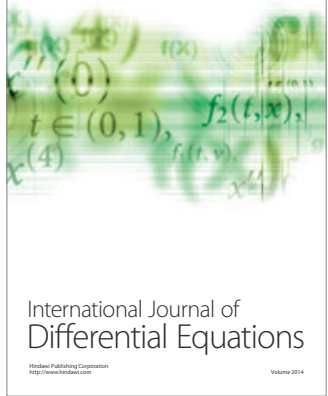
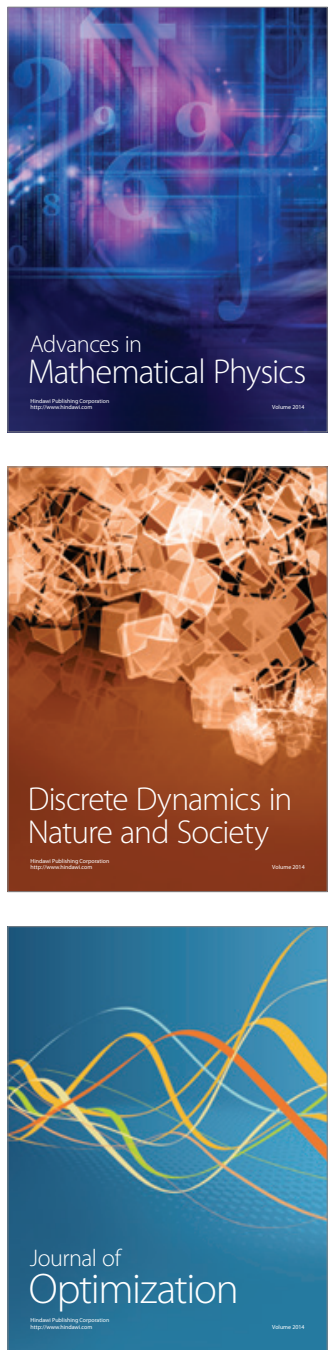